\title{
Analysing Ewald's Method for the Evaluation of Green's Functions
}

\author{
for Periodic Media
}

\author{
Tilo Arens*, Kai Sandfort ${ }^{\dagger}$ Susanne SchmitT ${ }^{\ddagger}$ \\ Karlsruhe Institute of Technology (KIT), 76128 Karlsruhe, Germany \\ and Armin LeChleiter ${ }^{\S}$
}

INRIA Saclay, Ecole Polytechnique, Route de Saclay, 91128 Palaiseau, France

Manuscript submitted for publication

\begin{abstract}
Expressions for periodic Green's functions for the Helmholtz equation in two and three dimensions are derived via Ewald's method. The decay rate of the series occurring in these expressions is analysed and rigorous estimates for the remainder are derived when the series are truncated and replaced by finite sums. The effect of choosing a control parameter occurring in Ewald's expressions is discussed and some recommendations for its choice are given based on the aforementioned estimates. We present various numerical examples for the resulting method for evaluating the Green's functions. The results can also be carried over to evaluating the partial derivatives.
\end{abstract}

\section{Introduction}

In this article, we carry out a theoretical and numerical analysis of the so-called Ewald's representation of the periodic and biperiodic Green's functions for the Helmholtz equation in two and three spatial

\footnotetext{
*Corresponding author. Email: arens@numathics.com

${ }^{\dagger}$ Email: kai.sandfort@kit.edu

${ }^{\ddagger}$ Email: susanne.schmitt@kit.edu

§Email: alechle@cmap.polytechnique.fr
} 
dimensions. There are applications for these functions from which integral equation formulations for scattering problems are of particular interest to the authors (Arens, 2010; Sandfort, 2010). The need to evaluate the Green's function and its derivatives is often a limiting factor in these approaches. For the Helmholtz operator and the free-space or half-space geometry, there are simple expressions for the Green's functions which can easily be evaluated. For more complicated geometries, including periodic media, the Green's functions are usually given formally by series expansions which might be only slowly convergent or even divergent depending on the values of the problem parameters. However, to implement efficient numerical schemes for solving the boundary integral equations, it is essential to have representations of the Green's functions available which allow the fast and accurate evaluation for all admissible problem parameters.

In the review article (Linton, 1998), a number of analytical techniques to derive such convenient expressions for the Green's function for the two-dimensional Helmholtz equation in periodic domains are discussed and compared in-depth. One of the best-performing methods presented is Ewald's transformation. This transformation was originally derived in (Ewald, 1921) for the computation of three-dimensional lattice potentials. It splits the series representation of the lattice potential, and likewise that of the periodic Green's function, into a sum of two series, of types different from that of the original series, which are both exponentially convergent. From the big amount of alternative methods, besides the references given in (Linton, 1998), we also mention (Sadov, 1997) and (Kurkcu \& Reitich, 2009).

Ewald's method became commonly used in the physics community and is covered predominantly in the engineering and physics literature, though it is attractive not only from the numerical, but also the theoretical point of view. In addition to the two-dimensional quasi-periodic Green's function as discussed in (Linton, 1998) and references given therein, expressions for the three-dimensional quasibiperiodic Green's function were also derived (Kambe, 1967). However, this function has not been discussed much further in the literature. For the two-dimensional quasi-periodic Green's function, Ewald's approach has been discussed in a number of articles, e.g. (Jordan et al., 1986) and (Capolino 
et al., 2005). In all cases known to the authors, asymptotical behaviour of the terms in the series is discussed and conclusions are backed by some numerical examples.

The present paper contributes to this subject firstly by giving, in Section 3, a detailed and rigorous derivation of the Ewald representation for the two-dimensional quasi-periodic Green's function for the Helmholtz equation, clearly separating technicalities from core arguments. A companion analysis along similar lines for the three-dimensional bi-quasi-periodic Green's function is contained in (Arens, 2010).

Secondly, and this is the main novel contribution, we give in Sections 4 and 5 rigorous and computable error bounds for truncated versions of the series both for the two- and the three-dimensional cases. Provided that cancellation effects do not play a dominant role, these estimates allow the evaluation of the Green's functions to a prescribed error.

A further aspect of Ewald's method is that it involves a parameter that can be used to influence the speed of convergence of all series. Various authors give recommendations for choosing this parameter (Jordan et al., 1986; Mathis \& Peterson, 1996; Kustepeli \& Martin, 2000; Capolino et al., 2005). The rule of thumb appears to be to choose a constant parameter that gives good numerical accuracy reliably. However, an optimal choice should minimise the total effort for the evaluation for a prescribed accuracy. We propose an algorithm that chooses this parameter automatically while guaranteeing optimality in some sense. As a bad choice of the control parameter will also lead to cancellation and instability, conditions are formulated that prevent this case. For the two-dimensional case, the only similar work in this respect appears to be (Capolino et al., 2005) and our results for the $2 \mathrm{D}$ case in this respect only differ in details. For the three-dimensional case, our results appear to be completely new. A number of numerical examples illustrate the performance of the proposed methods. The computations were carried out using the program library libewald written by the authors in the programming language $\mathrm{C}$. The source code of this library, which contains programs to produce all results presented in this paper, is available at http://libewald.sourceforge.net.

In many applications, including boundary integral equation methods, not only the Green's func- 
tion, but also its gradient is required. Therefore, in Section 7 we study the gradient of the Ewald's representation and corresponding error estimates. Some numerical results are also given.

\section{Periodic Green's Functions}

The propagation of time-harmonic acoustic waves with wave number $k>0$ is modelled by the Helmholtz equation

$$
\Delta u+k^{2} u=0
$$

Absorption in the medium can be taken into account by allowing wave numbers with $\arg (k) \in[0, \pi / 2)$.

The fundamental solution, or Green's function in free-field conditions, of (2.1) is given by

$$
\Phi(x, y)=\frac{\mathrm{i}}{4} H_{0}^{(1)}(k|x-y|), \quad x, y \in \mathbb{R}^{2}, \quad x \neq y
$$

in two spatial dimensions, where $H_{\nu}^{(1)}$ is the Hankel function of the first kind and of order $\nu$, and by

$$
\Phi(x, y)=\frac{1}{4 \pi} \frac{\mathrm{e}^{\mathrm{i} k|x-y|}}{|x-y|}, \quad x, y \in \mathbb{R}^{3}, \quad x \neq y
$$

in three spatial dimensions. Note that through

$$
\frac{\mathrm{e}^{\mathrm{i} k r}}{r}=\sqrt{\frac{k \pi}{2 r}} H_{-1 / 2}^{(1)}(k r), \quad r>0
$$

both fundamental solutions can be expressed via a Hankel function of the first kind.

Consider now a domain $\Omega \subseteq \mathbb{R}^{2}$ which is $L$-periodic, $L>0$, in the $x_{1}$-direction, i.e. for $x \in \Omega$ there holds $\left(x_{1}+\mu L, x_{2}\right)^{\top} \in \Omega$ for all $\mu \in \mathbb{Z}$. If (2.1) is considered in such a domain, it is usual to consider $\alpha$-quasi-periodic fields for some $\alpha \in \mathbb{R}$. Such fields are $L$-periodic up to a phase shift,

$$
u\left(x_{1}+L, x_{2}\right)=\mathrm{e}^{\mathrm{i} L \alpha} u\left(x_{1}, x_{2}\right), \quad x \in \Omega .
$$

A typical example of such a field is a plane wave $u(x)=\exp (i k d \cdot x)$ with $d$ a unit vector, which is $k d_{1}$-quasi-periodic.

In scattering problems for such $L$-periodic media, one considers the $\alpha$-quasi-periodic Green's function for the Helmholtz equation $G_{\mathrm{q} 2}$. One possible expression for this function is a series of 
translated point sources,

$$
G_{\mathrm{q} 2}(x-y)=\frac{\mathrm{i}}{4} \sum_{\mu \in \mathbb{Z}} \mathrm{e}^{\mathrm{i} \mu \alpha L} H_{0}^{(1)}\left(k\left|x-y-\mu\left(\begin{array}{l}
L \\
0
\end{array}\right)\right|\right),
$$

where $x, y \in \mathbb{R}^{2}$ such that $x-y \neq \mu(L, 0)^{\top}$ for all $\mu \in \mathbb{Z}$. Convergence of the series in (2.5) can be established from asymptotic decay rates of Hankel functions in the cases where

$$
\operatorname{Im}(k)>0 \quad \text { or } \quad\left[k>0 \quad \text { and } \quad \alpha+\frac{2 \pi}{L} \mu \neq k \quad \text { for all } \mu \in \mathbb{Z}\right]
$$

is satisfied (see e.g. (Arens, 1999) for explicit calculations). For real $k$, the terms in (2.5) decay as $|\mu|^{-1 / 2}$ so that convergence is rather slow and the series certainly does not converge absolutely. A faster converging representation of this Green's function is desirable for numerical purposes.

A further expression can be obtained by computing the expansion of $G_{\mathrm{q} 2}$ in trigonometric polynomials. We set

$$
\alpha_{\mu}=\frac{\alpha+2 \pi \mu / L}{k} \quad \text { and } \quad \beta_{\mu}=\sqrt{1-\alpha_{\mu}^{2}}, \quad \mu \in \mathbb{Z}
$$

where the square root function is analytically continued to the complex plane except for a branch cut along the negative imaginary axis. Then the Fourier series expansion of $G_{\mathrm{q} 2}$ is given by

$$
G_{\mathrm{q} 2}(x-y)=\frac{\mathrm{i}}{2 k L} \sum_{\mu \in \mathbb{Z}} \frac{1}{\beta_{\mu}} \mathrm{e}^{\mathrm{i} k\left[\alpha_{\mu}\left(x_{1}-y_{1}\right)+\beta_{\mu}\left|x_{2}-y_{2}\right|\right]},
$$

again for $x, y \in \mathbb{R}^{2}$ such that $x-y \neq \mu(L, 0)^{\top}$ for any $\mu \in \mathbb{Z}$. Expression (2.7) is exponentially convergent whenever $x_{2} \neq y_{2}$. However, for real $k$ and $x_{2}=y_{2}$ we observe a decay of $|\mu|^{-1}$ of the terms in the series so convergence is again slow. Also, if $2 \pi /(k L)$ is small, $\beta_{\mu}$ will be real for a large range of $\mu$ and the growth of its absolute value relatively slow outside this range so that many terms in (2.7) are required for an accurate numerical evaluation of $G_{\mathrm{q} 2}$.

The second Green's function under consideration is the three-dimensional analogue of $G_{\mathrm{q} 2}$. We consider a domain $\Omega \subseteq \mathbb{R}^{3}$ which is assumed to be $L_{1}$-periodic in the $x_{1}$-direction and $L_{2}$-periodic in the $x_{2}$-direction. For ease of notation, we define the lattice translation vectors $p^{(\mu)}$ and the reciprocal 
lattice vectors $q^{(\mu)}$ by

$$
p^{(\mu)}=\left(\begin{array}{c}
\mu_{1} L_{1} \\
\mu_{2} L_{2} \\
0
\end{array}\right) \quad \text { and } \quad q^{(\mu)}=\left(\begin{array}{c}
\mu_{1} 2 \pi / L_{1} \\
\mu_{2} 2 \pi / L_{2} \\
0
\end{array}\right), \quad \mu \in \mathbb{Z}^{2},
$$

respectively. Similarly as for the two-dimensional case, we call a field $u \alpha$-quasi-biperiodic for some $\alpha \in \mathbb{R}^{3}, \alpha_{3}=0$, if

$$
u\left(x+p^{(\mu)}\right)=\mathrm{e}^{\mathrm{i} \alpha \cdot p^{(\mu)}} u(x), \quad x \in \Omega, \quad \mu \in \mathbb{Z}^{2} .
$$

The $\alpha$-quasi-biperiodic Green's function can be expressed formally as (Arens, 2010; Kambe, 1967)

$$
G_{\mathrm{q} 3}(x-y)=\frac{1}{4 \pi} \sum_{\mu \in \mathbb{Z}^{2}} \mathrm{e}^{\mathrm{i} \alpha \cdot p^{(\mu)}} \frac{\mathrm{e}^{\mathrm{i} k\left|x-y-p^{(\mu)}\right|}}{\left|x-y-p^{(\mu)}\right|}
$$

for $x, y \in \Omega$ such that $x-y \neq p^{(\mu)}$ for any $\mu \in \mathbb{Z}^{2}$. Setting

$$
\alpha^{(\mu)}=\frac{\alpha+q^{(\mu)}}{k} \quad \text { and } \quad \rho_{\mu}=\sqrt{1-\left|\alpha^{(\mu)}\right|^{2}}, \quad \mu \in \mathbb{Z}^{2}
$$

an expansion in trigonometric polynomials with respect to both $x_{1}$ and $x_{2}$ gives

$$
G_{\mathrm{q} 3}(x-y)=\frac{\mathrm{i}}{2 k L_{1} L_{2}} \sum_{\mu \in \mathbb{Z}^{2}} \frac{1}{\rho_{\mu}} \mathrm{e}^{\mathrm{i} k\left[\alpha^{(\mu)} \cdot(\tilde{x}-\tilde{y})+\rho_{\mu}\left|x_{3}-y_{3}\right|\right]} .
$$

Here, and in all further arguments, we denote for $x \in \mathbb{R}^{3}$ by $\tilde{x}$ the projection onto the $x_{1}, x_{2}$ )-plane, i.e. $\tilde{x}=\left(x_{1}, x_{2}, 0\right)^{\top}$.

For such two-dimensional lattice sums, absolute convergence is required to make the expression well-defined. In (2.8), this is the case for $\operatorname{Im}(k)>0$, but not for a real wave number. The expression (2.9) is absolutely convergent even for real $k$, but $x_{3} \neq y_{3}$ is required. Hence, in the case of a biperiodic grating, an alternative exponentially convergent representation of the Green's function is not only desirable for numerical but also for analytical purposes.

\section{Ewald's Method}

The principle of Ewald's method for the derivation of quickly convergent representations of periodic Green's functions is rather simple. We heuristically present the idea for a function periodic in one 
direction. Consider a function $f: \Omega \rightarrow \mathbb{C}$ which has a singularity at 0 and which is slowly decaying for large arguments. Next, we define the $L$-periodic function

$$
F(x)=\sum_{\mu \in \mathbb{Z}} f\left(x+\mu L e_{1}\right), \quad x \in \Omega,
$$

where $e_{1}$ is the first coordinate unit vector. Of course, $\Omega$ is assumed to be a domain such that $x+\mu L e_{1} \in \Omega$ for all $x \in \Omega$ and all $\mu \in \mathbb{Z}$.

For both the Green's functions considered in Section 2, a respective $f$ will somehow involve a Hankel function. As will be shown below, we may split a Hankel function into a singular but quickly decaying and a smooth but slowly decaying function. In other words, we write $f=g+(f-g)$, where $g$ has the same singularity at 0 as $f$ and is exponentially decaying for large arguments, to obtain

$$
F(x)=\sum_{\mu \in \mathbb{Z}} g\left(x+\mu L e_{1}\right)+\sum_{\mu \in \mathbb{Z}}\left(f\left(x+\mu L e_{1}\right)-g\left(x+\mu L e_{1}\right)\right), \quad x \in \Omega .
$$

The second sum in this expression may be rewritten by means of the so-called Poisson summation formula, which yields

$$
F(x)=\sum_{\mu \in \mathbb{Z}} g\left(x+\mu L e_{1}\right)+\frac{1}{L} \sum_{\mu \in \mathbb{Z}}\left[\hat{f}\left(\frac{2 \pi \mu}{L}, P x\right)-\hat{g}\left(\frac{2 \pi \mu}{L}, P x\right)\right] \mathrm{e}^{\mathrm{i}(2 \pi \mu / L) x_{1}},
$$

for $x \in \Omega$, where $P x$ denotes the orthogonal projection of $x$ onto the orthogonal complement of $e_{1}$ and $\hat{f}$ denotes the one-dimensional Fourier transform in the direction of $\hat{p}$,

$$
\hat{f}(\xi, z)=\int_{-\infty}^{\infty} f\left(z+\eta e_{1}\right) \mathrm{e}^{-\mathrm{i} \xi \eta} \mathrm{d} \eta, \quad \xi \in \mathbb{R}, \quad z \in\left(\operatorname{span}\left\{e_{1}\right\}\right)^{\perp} .
$$

Now, both series appearing in (3.1) are quickly convergent: the function $g$ is exponentially decreasing itself while $f-g$ is smooth and hence its Fourier transform is quickly decaying for large argument.

Let us first work out the necessary expressions for $G_{\mathrm{q} 2}$ in detail. For the derivation, we will assume that $\arg (k) \in(0, \pi / 2)$. In the final result, it is then possible to carry out the $\operatorname{limit} \operatorname{Im}(k) \rightarrow 0$, so that the representation of the Green's function we obtain is valid for real $k$ as well.

As shown in detail in (Arens, 2010), though the result can be found in various places in the literature, from standard expressions for Bessel function one may derive the following representation 
for the Hankel function of the first kind and of order $\nu \in \mathbb{R}$,

$$
H_{\nu}^{(1)}(k x)=\frac{2}{\mathrm{i} \pi} \mathrm{e}^{-\mathrm{i} \pi \nu}\left(\frac{k}{2 x}\right)^{\nu} \int_{\gamma_{1}} t^{-2 \nu-1} \exp \left(-x^{2} t^{2}+\frac{k^{2}}{4 t^{2}}\right) \mathrm{d} t
$$

where $x>0$ and $\arg (k) \in(0, \pi / 2)$. Furthermore, $\gamma_{1}$ denotes an integration path in the complex plane that starts at the origin in the direction $\mathrm{e}^{-\mathrm{i} \pi / 4}$ and approaches infinity in the direction $\mathrm{e}^{\mathrm{i} \arg (k) / 2}$, see Figure 1.

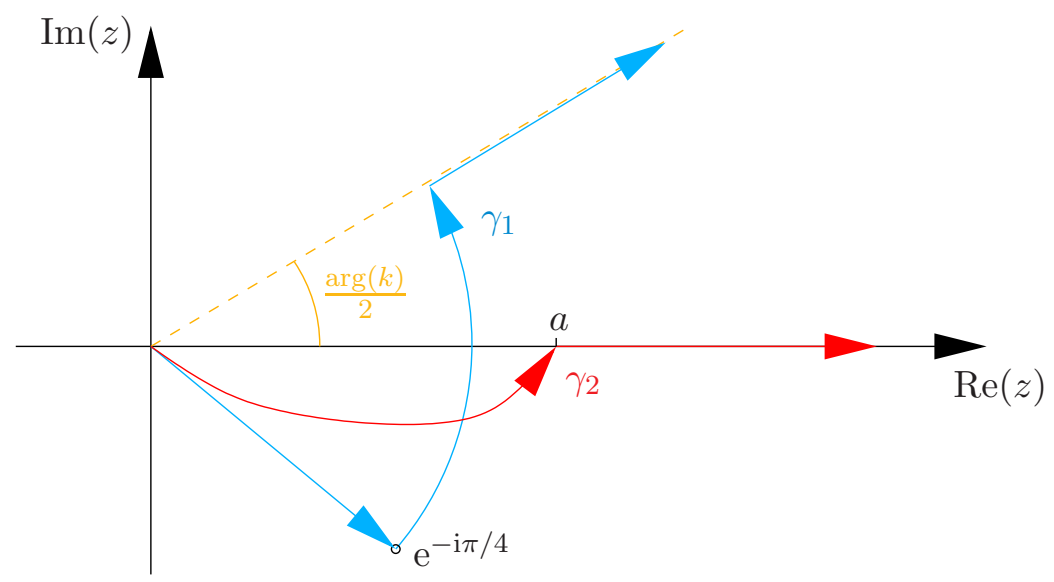

Figure 1: Contour paths $\gamma_{1}$ and $\gamma_{2}$.

The path of integration may deviate from $\gamma_{1}$ as long as two conditions are met: The direction at which the path leaves the origin must not change and the path must go to infinity at a direction $\mathrm{e}^{\mathrm{i} \varphi}$ with $-\pi / 4<\varphi<\pi / 4$. This ensures $\operatorname{Re}\left(t^{2}\right)>0$ so that the integrand decays exponentially for $|t| \rightarrow \infty$. For $\nu>-1 / 2$, we may even pass to the limit $\varphi \rightarrow-\pi / 4$, in which case the integral exists as an improper integral.

For the application of Ewald's method, we use a path that connects the origin with a point $a>0$ and then continues to infinity along the positive real axis. This integration contour is shown in Figure 1 as $\gamma_{2}$.

Let us define the function obtained by an integration along that section of $\gamma_{2}$ which coincides with the real axis,

$$
g_{\nu}(k x)=\frac{2}{\mathrm{i} \pi} \mathrm{e}^{-\mathrm{i} \pi \nu}\left(\frac{k}{2 x}\right)^{\nu} \int_{a}^{\infty} t^{-2 \nu-1} \exp \left(-x^{2} t^{2}+\frac{k^{2}}{4 t^{2}}\right) \mathrm{d} t .
$$


Note that $g_{\nu}$ is a continuous function of $k$ and well-defined also for $k>0$. For representing $G_{\mathrm{q} 2}$, we are dealing with the case $\nu=0$. We can show that $g_{0}$ has the same type of singularity at the origin as $H_{0}^{(1)}$ and decays exponentially for large argument.

Lemma 3.1 For $x>0$ and $\arg (k) \in[0, \pi / 2)$, we have

$$
g_{0}(k x)=\frac{1}{\mathrm{i} \pi}\left(-C-2 \ln (a x)-\sum_{n=1}^{\infty} \frac{(-1)^{n}}{n n !}(a x)^{2 n}\right) J_{0}(k x)+h\left(k x, \frac{k}{a}\right),
$$

where $h$ is an analytic function with respect to both arguments, and

$$
\left|g_{0}(k x)\right| \leq \frac{1}{\pi} \exp \left(\frac{|k|^{2}}{4 a^{2}}\right) \frac{\exp \left(-(a x)^{2}\right)}{(a x)^{2}} .
$$

Proof: Inserting the power series representation of the exponential function and using the substitution $s=t^{2} / a^{2}$, we obtain

$$
\begin{aligned}
g_{0}(k x) & =\frac{2}{\mathrm{i} \pi} \sum_{j=0}^{\infty} \frac{1}{j !}\left(\frac{k}{2}\right)^{2 j} \int_{a}^{\infty} t^{-2 j-1} \exp \left(-x^{2} t^{2}\right) \mathrm{d} t \\
& =\frac{1}{\mathrm{i} \pi} \sum_{j=0}^{\infty} \frac{1}{j !}\left(\frac{k}{2 a}\right)^{2 j} \int_{1}^{\infty} s^{-j-1} \exp \left(-(a x)^{2} s\right) \mathrm{d} s .
\end{aligned}
$$

We define the generalised exponential integral $I_{j}(\tau)=\int_{1}^{\infty} s^{-j-1} \mathrm{e}^{-\tau s} \mathrm{~d} s, j=0,1,2, \ldots$, and the coefficients

$$
c_{j, l}=\frac{(-1)^{l}}{\prod_{m=0}^{l-1}(j-m)}, \quad j=0,1,2, \ldots, \quad l=0, \ldots, j .
$$

Integration by parts yields

$$
I_{j+1}(\tau)=\frac{\mathrm{e}^{-\tau}}{j+1}-\frac{\tau}{j+1} I_{j}(\tau), \quad j=0,1,2, \ldots,
$$

and a simple induction argument leads to the explicit formula

$$
I_{j}(\tau)=c_{j, j} \tau^{j} I_{0}(\tau)-\left(\sum_{l=1}^{j} c_{j, l} \tau^{l-1}\right) \mathrm{e}^{-\tau}, \quad j=0,1,2, \ldots
$$

Hence

$$
g_{0}(k x)=\frac{1}{\mathrm{i} \pi}\left[I_{0}\left((a x)^{2}\right) \sum_{j=0}^{\infty} \frac{c_{j, j}}{j !}\left(\frac{k x}{2}\right)^{2 j}-\mathrm{e}^{-(a x)^{2}} \sum_{j=0}^{\infty} \sum_{l=1}^{j} \frac{c_{j, l}}{j !}\left(\frac{k}{2 a}\right)^{2 j}(a x)^{2(l-1)}\right]
$$


which we rewrite slightly as

$$
g_{0}(k x)=\frac{1}{\mathrm{i} \pi}\left[I_{0}\left((a x)^{2}\right) \sum_{j=0}^{\infty} \frac{c_{j, j}}{j !}\left(\frac{k x}{2}\right)^{2 j}-\mathrm{e}^{-(a x)^{2}} \sum_{l=0}^{\infty}\left(\frac{k x}{2}\right)^{2 l} \sum_{j=1}^{\infty} \frac{c_{j+l, l+1}}{(j+l) !}\left(\frac{k}{2 a}\right)^{2 j}\right] .
$$

Note that $c_{j, j}=(-1)^{j} / j !, j=0,1,2, \ldots$, so that we can substitute

$$
\sum_{j=0}^{\infty} \frac{c_{j, j}}{j !}\left(\frac{k x}{2}\right)^{2 j}=\sum_{j=0}^{\infty} \frac{(-1)^{j}}{(j !)^{2}}\left(\frac{k x}{2}\right)^{2 j}=J_{0}(k x)
$$

where $J_{0}$ denotes the Bessel function of order 0 (see (Abramowitz \& Stegun, 1965, formula 9.1.10)).

For $I_{0}$, we obtain from (Abramowitz \& Stegun, 1965, formulae 5.1.1 and 5.1.12)

$$
I_{0}(\tau)=E_{1}(\tau)=-C-\ln \tau-\sum_{n=1}^{\infty} \frac{(-1)^{n}}{n n !} \tau^{n},
$$

where $C$ is Euler's constant and $E_{1}$ denotes the exponential integral function. Hence

$$
\begin{aligned}
g_{0}(k x)=\frac{1}{\mathrm{i} \pi}\left[\left(-C-2 \ln (a x)-\sum_{n=1}^{\infty} \frac{(-1)^{n}}{n n !}(a x)^{2 n}\right)\right. & J_{0}(k x) \\
& \left.-\mathrm{e}^{-(a x)^{2}} \sum_{l=0}^{\infty}\left(\frac{k x}{2}\right)^{2 l} \sum_{j=1}^{\infty} \frac{c_{j+l, l+1}}{(j+l) !}\left(\frac{k}{2 a}\right)^{2 j}\right],
\end{aligned}
$$

which is the first part of the assertion.

For $j=0,1,2, \ldots$, we can further estimate

$$
\int_{1}^{\infty} s^{-j-1} \exp \left(-(a x)^{2} s\right) \mathrm{d} s \leq \int_{1}^{\infty} \exp \left(-(a x)^{2} s\right) \mathrm{d} s=\frac{\exp \left(-(a x)^{2}\right)}{(a x)^{2}}
$$

Thus,

$$
\left|g_{0}(k x)\right| \leq \frac{1}{\pi} \sum_{j=0}^{\infty} \frac{1}{j !}\left|\frac{k}{2 a}\right|^{2 j} \frac{\exp \left(-(a x)^{2}\right)}{(a x)^{2}}=\frac{1}{\pi} \exp \left(\frac{|k|^{2}}{4 a^{2}}\right) \frac{\exp \left(-(a x)^{2}\right)}{(a x)^{2}}
$$

and the proof is complete.

We now start from (2.5) replacing the Hankel function by $g_{0}$ and define for $z \in \mathbb{R}^{2}, z \neq 0$,

$$
\begin{aligned}
F_{\mathrm{q} 2}^{(1)}(z) & \left.=\frac{\mathrm{i}}{4} \sum_{\mu \in \mathbb{Z}} \mathrm{e}^{\mathrm{i} \mu \alpha L} g_{0}\left(k\left|z-\mu L e_{1}\right|\right)\right) \\
& =\sum_{\mu \in \mathbb{Z}} \frac{\mathrm{e}^{\mathrm{i} \mu \alpha L}}{4 \pi} \sum_{j=0}^{\infty} \frac{1}{j !}\left(\frac{k}{2 a}\right)^{2 j} \int_{1}^{\infty} s^{-j-1} \mathrm{e}^{-\left(a\left|z-\mu L e_{1}\right|\right)^{2} s} \mathrm{~d} s
\end{aligned}
$$


The heuristic arguments show a possibility to obtain a quickly convergent representation of

$$
F_{\mathrm{q} 2}^{(2)}(z):=G_{\mathrm{q} 2}(z)-F_{\mathrm{q} 2}^{(1)}(z)
$$

We set

$$
h_{0}(k x)=H_{0}^{(1)}(k x)-g_{0}(k x)=\frac{2}{\mathrm{i} \pi} \int_{\tilde{\gamma}_{2}} t^{-1} \exp \left(-x^{2} t^{2}+\frac{k^{2}}{4 t^{2}}\right) \mathrm{d} t
$$

where $\tilde{\gamma}_{2}$ denotes that part of $\gamma_{2}$ which is not the interval $(a, \infty)$. From the Fourier transform

$$
\int_{-\infty}^{\infty} \exp \left(-\eta^{2} t^{2}\right) \exp (-\mathrm{i} \xi \eta) \mathrm{d} \eta=\mathrm{e}^{-\xi^{2} /\left(4 t^{2}\right)} \int_{-\infty}^{\infty} \exp \left(-\left(\eta t+\frac{\mathrm{i} \xi}{2 t}\right)^{2}\right) \mathrm{d} \eta=\mathrm{e}^{-\xi^{2} /\left(4 t^{2}\right)} \frac{\sqrt{\pi}}{t}
$$

where the last equality follows from an application of Cauchy's integral theorem, we obtain

$$
\int_{-\infty}^{\infty} h_{0}(k|x|) \mathrm{e}^{-\mathrm{i} x_{1} s} \mathrm{~d} x_{1}=\frac{2}{\mathrm{i} \sqrt{\pi}} \int_{\tilde{\gamma}_{4}} t^{-2} \mathrm{e}^{-x_{2}^{2} t^{2}} \exp \left(\frac{k^{2}-s^{2}}{4 t^{2}}\right) \mathrm{d} t
$$

After compensating for the factor $\mathrm{e}^{\mathrm{i} \mu \alpha L}$, we can now apply Poisson's summation formula to conclude

$$
F_{\mathrm{q} 2}^{(2)}(z)=\frac{2}{\mathrm{i} \sqrt{\pi} L} \sum_{\mu \in \mathbb{Z}} \mathrm{e}^{\mathrm{i} k \alpha_{\mu} z_{1}} \int_{\tilde{\gamma}_{2}} t^{-2} \mathrm{e}^{-\left(z_{2}\right)^{2} t^{2}} \exp \left(\frac{k^{2} \beta_{\mu}^{2}}{4 t^{2}}\right) \mathrm{d} t .
$$

A further simplification of this expression can be achieved, starting from the substitution $u=1 / t$.

Setting $\gamma_{3}=\left\{z \in \mathbb{C}: u=1 / t, t \in \tilde{\gamma}_{2}\right\}$ gives

$$
F_{\mathrm{q} 2}^{(2)}(z)=\frac{2}{\mathrm{i} \sqrt{\pi} L} \sum_{\mu \in \mathbb{Z}} \mathrm{e}^{\mathrm{i} k \alpha_{\mu} z_{1}} \int_{\gamma_{3}} \exp \left(-\frac{z_{2}^{2}}{u^{2}}+\frac{u^{2} k^{2} \beta_{\mu}^{2}}{4}\right) \mathrm{d} u
$$

We have to distinguish cases depending on the sign of $\operatorname{Re}\left(k^{2} \beta_{\mu}^{2}\right)$. Suppose first that $\operatorname{Re}\left(k^{2} \beta_{\mu}^{2}\right)<0$. In this case, the path of integration can be shifted to the interval $(1 / a, \infty)$ and (Abramowitz \& Stegun, 1965, equation 7.4.33) yields

$$
\int_{\gamma_{3}} \mathrm{e}^{-\frac{z_{2}^{2}}{u^{2}}+\frac{u^{2} k^{2} \beta_{\mu}^{2}}{4}} \mathrm{~d} u=\frac{\mathrm{i} \sqrt{\pi}}{2 k \beta_{\mu}}\left(\mathrm{e}^{-\mathrm{i} k \beta_{\mu} z_{2}} \operatorname{erfc}\left(a z_{2}-\mathrm{i} \frac{k \beta_{\mu}}{2 a}\right)+\mathrm{e}^{\mathrm{i} k \beta_{\mu} z_{2}} \operatorname{erfc}\left(-a z_{2}-\mathrm{i} \frac{k \beta_{\mu}}{2 a}\right)\right) .
$$

In the case $\operatorname{Re}\left(k^{2} \beta_{\mu}^{2}\right)>0$ we perform another substitution $s=-\mathrm{i} u$ and then apply (Abramowitz \& Stegun, 1965, equation 7.4.33) to end up with the same result. The case $\operatorname{Re}\left(k^{2} \beta_{\mu}^{2}\right)=0$ is obtained from the other two by continuity. Concluding, we have obtained the expression

$$
F_{\mathrm{q} 2}^{(2)}(z)=\frac{\mathrm{i}}{4 L} \sum_{\mu \in \mathbb{Z}} \frac{1}{k \beta_{\mu}} \mathrm{e}^{\mathrm{i} k \alpha_{\mu} z_{1}}\left(\mathrm{e}^{-\mathrm{i} k \beta_{\mu} z_{2}} \operatorname{erfc}\left(a z_{2}-\mathrm{i} \frac{k \beta_{\mu}}{2 a}\right)+\mathrm{e}^{\mathrm{i} k \beta_{\mu} z_{2}} \operatorname{erfc}\left(-a z_{2}-\mathrm{i} \frac{k \beta_{\mu}}{2 a}\right)\right)
$$


for the function defined in (3.4). It will be beneficial for subsequent arguments to write this function as two separate series which differ only in the sign of some arguments,

$$
F_{\mathrm{q} 2}^{(2, \pm)}(z)=\frac{\mathrm{i}}{4 L} \sum_{\mu \in \mathbb{Z}} \frac{1}{k \beta_{\mu}} \mathrm{e}^{\mathrm{i} k \alpha_{\mu} z_{1}} \mathrm{e}^{\mp \mathrm{i} k \beta_{\mu} z_{2}} \operatorname{erfc}\left( \pm a z_{2}-\mathrm{i} \frac{k \beta_{\mu}}{2 a}\right)
$$

From Lemma 3.1, we see that the terms in the representation of $F_{\mathrm{q} 2}^{(1)}$ are exponentially decaying. We prove the same for $F_{\mathrm{q} 2}^{(2)}$.

Lemma 3.2 Choose $\mu_{0} \in \mathbb{Z}$ such that $\left|k \alpha_{\mu_{0}}\right| \leq\left|k \alpha_{\mu}\right|$ for all $\mu \in \mathbb{Z}$. Then there exists $M \in \mathbb{N}$ dependent on $k, \alpha$ and $z_{2}$ such that for all $\mu \in \mathbb{Z}$ with $|\mu| \geq M$,

$$
\left|\mathrm{e}^{\mp \mathrm{i} k \beta_{\mu_{0}+\mu} z_{2}} \operatorname{erfc}\left( \pm a z_{2}-\mathrm{i} \frac{k \beta_{\mu_{0}+\mu}}{2 a}\right)\right| \leq \exp \left(\frac{|k|^{2}}{4 a^{2}}-a^{2} z_{2}^{2}-\frac{\pi^{2}}{(a L)^{2}}(|\mu|-1)^{2}\right) .
$$

Proof: The complementary error function can be expressed using the Faddeeva function $w$ (see (Abramowitz \& Stegun, 1965, Formula 7.1.3)). Thus, the terms in the expression for $F_{\mathrm{q} 2}^{(2)}$ can be written as

$$
\mathrm{e}^{\mp \mathrm{i} k \beta_{\mu} z_{2}} \operatorname{erfc}\left( \pm a z_{2}-\mathrm{i} \frac{k \beta_{\mu}}{2 a}\right)=\mathrm{e}^{-a^{2} z_{2}^{2}+\left(\frac{k \beta_{\mu}}{2 a}\right)^{2}} w\left( \pm \mathrm{i} a z_{2}+\frac{k \beta_{\mu}}{2 a}\right)
$$

If the absolute value of $\mu$ is large enough, we have $\arg \left( \pm \mathrm{i} a z_{2}+k \beta_{\mu} /(2 a)\right) \in[0, \pi / 2)$. For such arguments, the absolute value of the Faddeeva function is bounded by 1 . Indeed, for $x \in \mathbb{R}, y \in \mathbb{R}_{>0}$,

$$
\begin{aligned}
|w(x+\mathrm{i} y)|^{2} & =\frac{4}{\pi} \mathrm{e}^{-2 x^{2}+2 y^{2}}\left|\frac{2}{\sqrt{\pi}} \int_{y-\mathrm{i} x}^{\infty} \mathrm{e}^{-t^{2}} \mathrm{~d} t\right|^{2}=\frac{4}{\pi} \mathrm{e}^{-2 x^{2}+2 y^{2}}\left|\int_{0}^{\infty} \mathrm{e}^{-(s+y-\mathrm{i} x)^{2}} \mathrm{~d} s\right|^{2} \\
& =\frac{4}{\pi}\left|\int_{0}^{\infty} \mathrm{e}^{-2 s(y-\mathrm{i} x)-s^{2}} \mathrm{~d} s\right|^{2}=\frac{4}{\pi}\left|\int_{0}^{\infty} \int_{0}^{\infty} \mathrm{e}^{-2(s+t) y+2 \mathrm{i}(s-t) x-s^{2}-t^{2}} \mathrm{~d} s \mathrm{~d} t\right| \\
& \leq \frac{4}{\pi} \int_{0}^{\infty} \int_{0}^{\infty} \mathrm{e}^{-s^{2}-t^{2}} \mathrm{~d} s \mathrm{~d} t=1 .
\end{aligned}
$$

We further have for $\mu \in \mathbb{Z} \backslash\{0\}$ and $s \in\{-1,0,1\}$

$$
\left(\alpha+\frac{2 \pi}{L}\left(\mu_{0}+\mu\right)\right)^{2} \geq \frac{4 \pi}{L}\left(\alpha+\frac{2 \pi}{L}\left(\mu_{0}+s\right)\right)(\mu-s)+\frac{4 \pi^{2}}{L^{2}}(\mu-s)^{2}
$$

and hence

$$
\left(\alpha+\frac{2 \pi}{L}\left(\mu_{0}+\mu\right)\right)^{2} \geq \frac{4 \pi^{2}}{L^{2}}(|\mu|-1)^{2} .
$$


As

$$
k^{2} \beta_{\mu+\mu_{0}}^{2}=k^{2}-\left(\alpha+\frac{2 \pi}{L}\left(\mu_{0}+\mu\right)\right)^{2}
$$

combining the above estimate with (3.5) and the bound for the Faddeeva function yields the assertion.

The statements made in Lemmas 3.1 and 3.2 have been observed frequently in the literature, most often expressed using asymptotic formulae. Although these estimates give the qualitative behaviour of the series obtained by Ewald's method, they are not enough, however, to guarantee a given error tolerance in the numerical evaluation.

\section{Numerical Evaluation of the Ewald Representation}

It is the pupose of this section to analyse the difference between the partial sums

$$
\begin{aligned}
& F_{\mathrm{q} 2, M}^{(1)}(z)=\sum_{\mu=\mu_{0}-M}^{\mu_{0}+M} \frac{\mathrm{e}^{\mathrm{i} \mu \alpha L}}{4 \pi} \sum_{j=0}^{\infty} \frac{1}{j !}\left(\frac{k}{2 a}\right)^{2 j} \int_{1}^{\infty} s^{-j-1} \mathrm{e}^{-\left(a\left|z-\mu L e_{1}\right|\right)^{2} s} \mathrm{~d} s, \\
& F_{\mathrm{q} 2, M}^{(2, \pm)}(z)=\frac{\mathrm{i}}{4 L} \sum_{\mu=\mu_{0}-M}^{\mu_{0}+M} \frac{1}{k \beta_{\mu}} \mathrm{e}^{\mathrm{i} k \alpha_{\mu} z_{1}} \mathrm{e}^{\mp \mathrm{i} k \beta_{\mu} z_{2}} \operatorname{erfc}\left( \pm a z_{2}-\mathrm{i} \frac{k \beta_{\mu}}{2 a}\right),
\end{aligned}
$$

and the full series, respectively, and to discuss the computation of numeric values for these series. The parameter $\mu_{0}$ should be chosen to center the corresponding partial sum on the dominating terms in the series. Our results below will include reasonable choices.

We first address the series remaining in (4.1). Defining

$$
F_{\text {aux }}^{(1)}(R, \kappa, \nu)=\sum_{j=0}^{\infty} \frac{1}{j !} \kappa^{j} \int_{1}^{\infty} s^{-j-\nu} \mathrm{e}^{-R s} \mathrm{~d} s .
$$

the first function can be rewritten as

$$
F_{\mathrm{q} 2, M}^{(1)}(z)=\sum_{\mu=-M}^{M} \frac{\mathrm{e}^{\mathrm{i} \mu \alpha L}}{4 \pi} F_{\mathrm{aux}}^{(1)}\left(a^{2}\left|z-\mu L e_{1}\right|^{2},\left(\frac{k}{2 a}\right)^{2}, 1\right) .
$$

For most of the remaining discussion we will assume that we can evaluate $F_{\text {aux }}^{(1)}$ exactly, just as any other standard or special function used in our expressions. However, the following lemma gives us some essential properties of this function. 
Lemma 4.1 For $R>0$, arg $(\kappa) \in[0, \pi / 2)$ and $\nu \geq 0$ we have

$$
\left|F_{\text {aux }}^{(1)}(R, \kappa, \nu)\right| \leq \frac{\exp (|\kappa|-R)}{R}
$$

and additionally for all $M \in \mathbb{N}, \nu>0$,

$$
\left|F_{\text {aux }}^{(1)}(R, \kappa, \nu)-\sum_{j=0}^{M} \frac{1}{j !} \kappa^{j} \int_{1}^{\infty} s^{-j-\nu} \mathrm{e}^{-R s} \mathrm{~d} s\right| \leq \frac{\mathrm{e}^{|\kappa|-R}}{M+\nu}\left(\frac{|\kappa|}{M+1}\right)^{M+1} .
$$

Proof: The first estimate is elementary. The estimate for the remainder can be obtained from the observation that the integral in the expression for $F_{\text {aux }}^{(1)}$ is equal to a generalised exponential integral. From (Hopf, 1934, page 26) we obtain

$$
\frac{\mathrm{e}^{-R}}{R+p}<\int_{1}^{\infty} s^{-p} \mathrm{e}^{-R s} \mathrm{~d} s \leq \frac{\mathrm{e}^{-R}}{R+p-1}, \quad R, p>0 .
$$

Hence

$$
\left|\sum_{j=M+1}^{\infty} \frac{1}{j !} \kappa^{j} \int_{1}^{\infty} s^{-j-\nu} \mathrm{e}^{-R s} \mathrm{~d} s\right| \leq \frac{\mathrm{e}^{-R}}{R+M+\nu} \sum_{j=M+1}^{\infty} \frac{1}{j !}|\kappa|^{j} \leq \frac{\mathrm{e}^{|\kappa|-R}}{M+\nu}\left(\frac{|\kappa|}{M+1}\right)^{M+1} .
$$

It is now fairly simple to estimate the remainder for $F_{\mathrm{q} 2}^{(1)}$.

Theorem 4.2 Define $\mu_{0} \in \mathbb{Z}$ by $z_{1}-\mu_{0} L \in(-L / 2, L / 2]$ and let $M \in \mathbb{N}$. Then

$$
\left|F_{\mathrm{q} 2}^{(1)}(z)-F_{\mathrm{q} 2, M}^{(1)}(z)\right| \leq \frac{\exp \left(|k|^{2} /\left(4 a^{2}\right)-a^{2} z_{2}^{2}\right)}{2 \pi a^{2}\left(z_{2}^{2}+(M L)^{2}\right)\left(1-\exp \left(-2 a^{2} M L^{2}\right)\right)} \mathrm{e}^{-(a M L)^{2}}
$$

Proof: From the first estimate in Lemma 4.1 we have

$$
\begin{aligned}
\left|F_{\mathrm{q} 2}^{(1)}(z)-F_{\mathrm{q} 2, M}^{(1)}(z)\right| & \leq \frac{1}{4 \pi} \sum_{\left|\mu-\mu_{0}\right| \geq M+1}\left|F_{\mathrm{aux}}^{(1)}\left(a^{2}\left(\left(z_{1}-\mu L\right)^{2}+z_{2}^{2}\right),\left(\frac{k}{2 a}\right)^{2}, 1\right)\right| \\
& \leq \frac{\exp \left(\left|k^{2}\right| /\left(4 a^{2}\right)-a^{2} z_{2}^{2}\right)}{4 \pi} \sum_{\left|\mu-\mu_{0}\right| \geq M+1} \frac{\exp \left(-a^{2}\left(z_{1}-\mu L\right)^{2}\right)}{a^{2}\left(\left(z_{1}-\mu L\right)^{2}+z_{2}^{2}\right)} .
\end{aligned}
$$

For $\left|\mu-\mu_{0}\right| \geq M+1$, we find

$$
\left(z_{1}-\mu L\right)^{2} \geq\left(z_{1}-\mu_{0} L \pm(M+1) L\right)^{2}+2 L\left(z_{1}-\mu_{0} \pm(M+1) L\right)\left(\mu_{0}-\mu \mp(M+1)\right),
$$


from which we conclude

$$
\left(z_{1}-\mu L\right)^{2} \geq M^{2} L^{2}+2 M L^{2}\left(\left|\mu_{0}-\mu\right|-(M+1)\right) \geq M^{2} L^{2} .
$$

Thus

$$
\sum_{\left|\mu-\mu_{0}\right| \geq M+1} \frac{\exp \left(-a^{2}\left(z_{1}-\mu L\right)^{2}\right)}{a^{2}\left(\left(z_{1}-\mu L\right)^{2}+z_{2}^{2}\right)} \leq \frac{2 \exp \left(-(a M L)^{2}\right)}{a^{2}\left((M L)^{2}+z_{2}^{2}\right)} \sum_{\mu=M+1}^{\infty} \mathrm{e}^{-2 M L^{2} a^{2}(\mu-(M+1))} .
$$

The assertion now follows using the geometric series.

The analysis of the two functions representing $F_{\mathrm{q} 2}^{(2)}$ is slightly more complicated. One way to evaluate the complementary error function is through (3.5) using the Faddeeva function. However, in the case $\pm a z_{2}+\operatorname{Im}\left(k \beta_{\mu} /(2 a)\right)<0$ a numerical evaluation of the right-hand side of (3.5) may not be stable. In this case, we use the elementary formula

$$
w(\bar{z})=\overline{w(-z)}=2 \mathrm{e}^{-\bar{z}^{2}}-\overline{w(z)}
$$

to obtain

$$
\mathrm{e}^{-\mathrm{i} k \beta_{\mu} z_{2}} \operatorname{erfc}\left(a z_{2}-\mathrm{i} \frac{k \beta_{\mu}}{2 a}\right)=2 \mathrm{e}^{-\mathrm{i} k \beta_{\mu} z_{2}}-\mathrm{e}^{-a^{2} z_{2}^{2}+\left(\frac{k \beta_{\mu}}{2 a}\right)^{2}} \overline{w\left(-\mathrm{i} a z_{2}+\frac{\overline{k \beta_{\mu}}}{2 a}\right)} .
$$

The representations (3.5) and (4.3) also lead to separate error estimates valid for different ranges of cut-off indices.

Theorem 4.3 Choose $\mu_{0}$ as in Lemma 3.2 and set $M_{0}=\left|k L \alpha_{\mu_{0}} /(2 \pi)\right|$. Then, for $M \geq M_{0}$ satisfying also $\min \operatorname{Im}\left(k \beta_{\mu_{0} \pm M}\right)>0$ and $\pm a z_{2}+\operatorname{Im}\left(k \beta_{\mu_{0}+M} /(2 a)\right) \geq 0$,

$$
\left|F_{\mathrm{q} 2}^{(2, \pm)}(z)-F_{\mathrm{q} 2, M}^{(2, \pm)}(z)\right| \leq \frac{\exp \left(\left|\frac{k}{2 a}\right|^{2}-a^{2} z_{2}^{2}-\frac{2 \pi^{2}\left(M-M_{0}\right)}{a^{2} L^{2}}\right)}{2 L \min \operatorname{Im}\left(k \beta_{\mu_{0} \pm M}\right)\left(1-\exp \left(-\frac{2 \pi^{2}\left(M-M_{0}\right)}{a^{2} L^{2}}\right)\right)} \mathrm{e}^{-\frac{\pi^{2}}{a^{2} L^{2}}\left(M-M_{0}\right)^{2}} .
$$

Proof: Recall that

$$
\left(k \beta_{\mu}\right)^{2}=k^{2}-\left(\alpha+\frac{2 \pi \mu}{L}\right)^{2}
$$

With arguments similar to those at the end of the proof of Lemma 3.2, we obtain for any integer $M \geq M_{0}$ and any $\mu \geq 0$,

$$
\left(\alpha+\frac{2 \pi\left(\mu_{0} \pm(M+\mu)\right)}{L}\right)^{2} \geq \frac{4 \pi^{2}}{L^{2}}\left[M-M_{0}+\mu\right]^{2} \geq \frac{4 \pi^{2}}{L^{2}}\left[\left(M-M_{0}\right)^{2}+2\left(M-M_{0}\right) \mu\right] .
$$


Hence, from (3.5) and the bound on the Faddeeva function,

$$
\begin{aligned}
\left|F_{\mathrm{q} 2}^{(2, \pm)}(z)-F_{\mathrm{q} 2, M}^{(2, \pm)}(z)\right| & =\frac{1}{4 L}\left|\sum_{\left|\mu-\mu_{0}\right|>M} \frac{1}{k \beta_{\mu}} \mathrm{e}^{\mathrm{i} k \alpha_{\mu} z_{1}} \mathrm{e}^{\mp \mathrm{i} k \beta_{\mu} z_{2}} \operatorname{erfc}\left( \pm a z_{2}-\mathrm{i} \frac{k \beta_{\mu}}{2 a}\right)\right| \\
& \leq \frac{\exp \left(\left|\frac{k}{2 a}\right|^{2}-a^{2} z_{2}^{2}\right)}{4 L} \sum_{\left|\mu-\mu_{0}\right|>M} \frac{1}{\left|k \beta_{\mu}\right|} \exp \left(-\frac{(\alpha+2 \pi \mu / L)^{2}}{4 a^{2}}\right) \\
& \leq \frac{\exp \left(\left|\frac{k}{2 a}\right|^{2}-a^{2} z_{2}^{2}-\frac{\pi^{2}\left(M-M_{0}\right)^{2}}{a^{2} L^{2}}\right)}{2 L \min \operatorname{Im}\left(k \beta_{\mu_{0} \pm M}\right)} \sum_{\mu=1}^{\infty} \exp \left(-\frac{2 \pi^{2}\left(M-M_{0}\right)}{a^{2} L^{2}} \mu\right) \\
& =\frac{\exp \left(\left|\frac{k}{2 a}\right|^{2}-a^{2} z_{2}^{2}-\frac{\pi^{2}\left(M-M_{0}\right)^{2}}{a^{2} L^{2}}\right)}{2 L \min \operatorname{Im}\left(k \beta_{\mu_{0} \pm M}\right)} \frac{\exp \left(-\frac{2 \pi^{2}\left(M-M_{0}\right)}{a^{2} L^{2}}\right)}{1-\exp \left(-\frac{2 \pi^{2}\left(M-M_{0}\right)}{a^{2} L^{2}}\right)} .
\end{aligned}
$$

In the case $\pm a z_{2}+\operatorname{Im}\left(k \beta_{\mu_{0}+M} /(2 a)\right)<0$, we require some additional estimates. For any $\mu \in \mathbb{Z}$ such that $k^{2} \alpha_{\mu}^{2} \geq \operatorname{Re}\left(k^{2}\right)$ we have

$$
\left|k \beta_{\mu}\right|^{2}=\left|k^{2} \beta_{\mu}^{2}\right| \geq\left|\operatorname{Re}\left(k^{2} \beta_{\mu}^{2}\right)\right|=\left|\operatorname{Re}\left(k^{2}\right)-k^{2} \alpha_{\mu}^{2}\right|=k^{2} \alpha_{\mu}^{2}-\operatorname{Re}\left(k^{2}\right)
$$

Choose $\mu_{0}$ and $M_{0}$ as in Lemma 3.2 and Theorem 4.3 and set

$$
M_{1}=M_{0}+ \begin{cases}\frac{L}{2 \pi} \sqrt{\operatorname{Re}\left(k^{2}\right)}, & \operatorname{Re}\left(k^{2}\right) \geq 0, \\ 0, & \text { otherwise. }\end{cases}
$$

Then for $\left|\mu-\mu_{0}\right|>M_{1}$ and if also $k^{2} \alpha_{\mu}^{2} \geq \operatorname{Re}\left(k^{2}\right)$, using (4.4),

$$
\begin{aligned}
\left|k \beta_{\mu}\right|^{2} \geq\left(\frac{2 \pi}{L}\right. & \left.\left(\left|\mu-\mu_{0}\right|-M_{1}\right)+\frac{2 \pi}{L} M_{1}-\left|\alpha+\frac{2 \pi}{L} \mu_{0}\right|\right)^{2}-\operatorname{Re}\left(k^{2}\right) \\
& \geq \frac{4 \pi^{2}}{L^{2}}\left(\left|\mu-\mu_{0}\right|-M_{1}\right)^{2}+\frac{4 \pi^{2}}{L^{2}}\left(M_{1}-M_{0}\right)^{2}-\operatorname{Re}\left(k^{2}\right) \geq \frac{4 \pi^{2}}{L^{2}}\left(\left|\mu-\mu_{0}\right|-M_{1}\right)^{2}
\end{aligned}
$$

Note furthermore that $k^{2} \alpha_{\mu}^{2} \geq \operatorname{Re}\left(k^{2}\right)$ if and only if $\operatorname{Re}\left(k \beta_{\mu}\right) \leq \operatorname{Im}\left(k \beta_{\mu}\right)$.

Theorem 4.4 Choose $\mu_{0}, M_{0}$ and $M_{1}$ as above. Then, for $M \geq M_{1}$ satisfying also $\operatorname{Im}\left(k \beta_{\mu_{0} \pm M}\right) \geq$ $\operatorname{Re}\left(k \beta_{\mu_{0} \pm M}\right)$,

$$
\begin{aligned}
\left|F_{\mathrm{q} 2}^{(2, \pm)}(z)-F_{\mathrm{q} 2, M}^{(2, \pm)}(z)\right| \leq & \frac{\exp \left(\left|\frac{k}{2 a}\right|^{2}-a^{2} z_{2}^{2}-\frac{2 \pi^{2}\left(M-M_{0}\right)}{a^{2} L^{2}}\right)}{2 L \min \operatorname{Im}\left(k \beta_{\mu_{0} \pm M}\right)\left(1-\exp \left(-\frac{2 \pi^{2}\left(M-M_{0}\right)}{a^{2} L^{2}}\right)\right)} \mathrm{e}^{-\frac{\pi^{2}}{a^{2} L^{2}}\left(M-M_{0}\right)^{2}} \\
& +\frac{1}{L \min \operatorname{Im}\left(k \beta_{\mu_{0} \pm M}\right)} \frac{\mathrm{e}^{-\frac{2 \pi\left|z_{2}\right|}{\sqrt{2} L}}}{1-\mathrm{e}^{-\frac{2 \pi\left|z_{2}\right|}{\sqrt{2} L}}} \mathrm{e}^{-\frac{2 \pi}{\sqrt{2} L}\left(M-M_{1}\right)\left|z_{2}\right|} .
\end{aligned}
$$


Proof: From (4.5) and the assumptions on $M$, we have for $\mu>0$,

$$
\left(\operatorname{Im}\left(k \beta_{\mu_{0} \pm(M+\mu)}\right)\right)^{2} \geq \frac{1}{2}\left|k \beta_{\mu_{0} \pm(M+\mu)}\right|^{2} \geq \frac{2 \pi^{2}}{L^{2}}\left(M+\mu-M_{1}\right)^{2} .
$$

Denote by $\mathcal{M}$ the set of those $\mu \in \mathbb{Z}$ for which $\pm a z_{2}+\operatorname{Im}\left(k \beta_{\mu} /(2 a)\right)<0$. Then, using (3.5) and $(4.3)$,

$$
\begin{aligned}
& \left|\sum_{\left|\mu-\mu_{0}\right|>M} \frac{1}{k \beta_{\mu}} \mathrm{e}^{\mathrm{i} k \alpha_{\mu} z_{1}} \mathrm{e}^{\mathrm{i} k \beta_{\mu} z_{2}} \operatorname{erfc}\left(-a z_{2}-\mathrm{i} \frac{k \beta_{\mu}}{2 a}\right)\right| \\
& \leq \sum_{\substack{\left|\mu-\mu_{0}\right|>M \\
\mu \in \mathcal{M}}} \frac{2}{\left|k \beta_{\mu}\right|} \mathrm{e}^{-\operatorname{Im}\left(k \beta_{\mu}\right)\left|z_{2}\right|}+\mathrm{e}^{\left|\frac{k}{2 a}\right|^{2}-a^{2} z_{2}^{2}} \sum_{\left|\mu-\mu_{0}\right|>M} \frac{1}{\left|k \beta_{\mu}\right|} \mathrm{e}^{-\frac{(\alpha+2 \pi \mu / L)^{2}}{4 a^{2}}} \\
& \leq \frac{4 \mathrm{e}^{-\frac{2 \pi}{\sqrt{2} L}\left(M-M_{1}\right)\left|z_{2}\right|}}{\min \operatorname{Im}\left(k \beta_{\mu_{0} \pm M}\right)} \frac{\mathrm{e}^{-\frac{2 \pi\left|z_{2}\right|}{\sqrt{2} L}}}{1-\mathrm{e}^{-\frac{2 \pi\left|z_{2}\right|}{\sqrt{2} L}}}+\frac{2 \mathrm{e}^{\left|\frac{k}{2 a}\right|^{2}-a^{2} z_{2}^{2}-\frac{\pi^{2}}{a^{2} L^{2}}\left(M-M_{0}\right)^{2}}}{\min \operatorname{Im}\left(k \beta_{\mu_{0} \pm M}\right)} \frac{\mathrm{e}^{-\frac{2 \pi^{2}}{a^{2} L^{2}}\left(M-M_{0}\right)}}{1-\mathrm{e}^{-\frac{2 \pi^{2}}{a^{2} L^{2}}\left(M-M_{0}\right)}} .
\end{aligned}
$$

By Theorem 4.3, the final estimate holds for $\pm a z_{2}+\operatorname{Im}\left(k \beta_{\mu} /(2 a)\right) \geq 0$ as well.

The estimate in Theorem 4.3 corresponds to the asymptotic decay rate of the terms in the series. The bound in Theorem 4.4 contains an additional term which decays at a slower rate. However, particularly in cases where $a z_{2}$ is negative and has small absolute value, only using Theorem 4.3 for deciding how many terms in the series to use for the approximation may lead to an unnecessarily large number of terms being used.

\section{The Three-Dimensional Setting}

Similarly as for the case of the quasi-periodic Green's function in two dimensions, Ewald's method may be applied to derive expressions for the quasi-biperiodic Green's function in three dimensions. The derivation, starting from (2.8), is similar to that in the two-dimensional case and it is carried out in (Arens, 2010) in detail. We obtain

$$
G_{\mathrm{q} 3}(z)=F_{\mathrm{q} 3}^{(1)}(z)+F_{\mathrm{q} 3}^{(2)}(z), \quad z \in \mathbb{R}^{3} \backslash\{0\},
$$


where

$$
\begin{aligned}
F_{\mathrm{q} 3}^{(1)}(z) & =\frac{a}{4 \pi^{3 / 2}} \sum_{\mu \in \mathbb{Z}^{2}} \mathrm{e}^{\mathrm{i} \alpha \cdot p^{(\mu)}} \sum_{j=0}^{\infty} \frac{1}{j !}\left(\frac{k}{2 a}\right)^{2 j} \int_{1}^{\infty} s^{-j-1 / 2} \mathrm{e}^{-a^{2}\left|z-p^{(\mu)}\right|^{2} s} \mathrm{~d} s, \\
F_{\mathrm{q} 3}^{(2, \pm)}(z) & =\frac{\mathrm{i}}{4 L_{1} L_{2}} \sum_{\mu \in \mathbb{Z}^{2}} \frac{1}{k \rho_{\mu}} \mathrm{e}^{\mathrm{i} k \alpha^{(\mu)} \cdot z} \mathrm{e}^{\mp \mathrm{i} k \rho_{\mu} z_{3}} \operatorname{erfc}\left( \pm a z_{3}-\mathrm{i} \frac{k \rho_{\mu}}{2 a}\right) .
\end{aligned}
$$

The equivalent of Lemma 3.1 is also shown in (Arens, 2010). The partial sums used for the evaluation will be written in the form

$$
\begin{aligned}
& F_{\mathrm{q} 3, M}^{(1)}(z)=\frac{a}{4 \pi^{3 / 2}} \sum_{m=0}^{M} \sum_{\left|\mu-\mu_{0}\right|_{\infty}=m} \frac{\mathrm{e}^{\mathrm{i} \alpha \cdot p^{(\mu)}}}{4 \pi} F_{\text {aux }}^{(1)}\left(a^{2}\left|z-p^{(\mu)}\right|^{2},\left(\frac{k}{2 a}\right)^{2}, \frac{1}{2}\right), \\
& F_{\mathrm{q} 3, M}^{(2, \pm)}(z)=\frac{\mathrm{i}}{4 L_{1} L_{2}} \sum_{m=0}^{M} \sum_{\left|\mu-\mu_{0}\right|_{\infty}=m} \frac{1}{k \rho_{\mu}} \mathrm{e}^{\mathrm{i} k \alpha^{(\mu)} \cdot z} \mathrm{e}^{\mp \mathrm{i} k \rho_{\mu} z_{3}} \operatorname{erfc}\left( \pm a z_{3}-\mathrm{i} \frac{k \rho_{\mu}}{2 a}\right) .
\end{aligned}
$$

Estimates for the remainders in the series can be obtained in a similar way to that used for the two-dimensional case. For their formulation, we will abbreviate $L=\min _{j=1,2} L_{j}$.

Theorem 5.1 Define $\mu_{0} \in \mathbb{Z}^{2}$ by $z-p^{\left(\mu_{0}\right)} \in\left(-L_{1} / 2, L_{1} / 2\right] \times\left(-L_{2} / 2, L_{2} / 2\right] \times \mathbb{R}$ and let $M \in \mathbb{N}$. Then

$$
\left|F_{\mathrm{q} 3}^{(1)}(z)-F_{\mathrm{q} 3, M}^{(1)}(z)\right| \leq \frac{8 \exp \left(|k|^{2} /\left(4 a^{2}\right)-a^{2} z_{3}^{2}\right)}{\pi^{3 / 2} a M L^{2}\left(1-\exp \left(-2 a^{2} M L^{2}\right)\right)} \mathrm{e}^{-(a M L)^{2}} .
$$

Proof: From Lemma 4.1 we have

$$
\left|F_{\mathrm{q} 3}^{(1)}(z)-F_{\mathrm{q} 3, M}^{(1)}(z)\right| \leq \frac{a \exp \left(\left|k^{2}\right| /\left(4 a^{2}\right)-a^{2} z_{3}^{2}\right)}{4 \pi^{3 / 2}} \sum_{m=M+1}^{\infty} \sum_{|\mu|_{\infty}=m} \frac{\exp \left(-a^{2}\left|\tilde{z}-p^{(\mu)}\right|^{2}\right)}{a^{2}\left|z-p^{(\mu)}\right|^{2}} .
$$

For $\mu \in \mathbb{Z}^{2}$ such that $\left|\mu-\mu_{0}\right|_{\infty}=m$ we choose $j_{0} \in\{1,2\}$ such that $\left|\mu_{j_{0}}-\mu_{0, j_{0}}\right|=m$. As in the proof of Theorem 4.2, we can then estimate for $\left|\mu-\mu_{0}\right|_{\infty}=m \geq M+1$,

$$
\left|\tilde{z}-p^{(\mu)}\right|^{2} \geq\left(z_{j_{0}}-\mu_{j_{0}} L_{j_{0}}\right)^{2} \geq M^{2} L^{2}+2 M L^{2}(m-(M+1)) \geq M^{2} L^{2} .
$$

We also have

$$
\left|z-p^{(\mu)}\right|^{2} \geq\left(z_{j_{0}}-\mu_{j_{0}} L_{j_{0}}\right)^{2} \geq\left(\mu_{j_{0}}-\mu_{0, j_{0}}\right)^{2}\left(L_{j_{0}}-\frac{z_{j_{0}}-\mu_{0, j_{0}} L_{j_{0}}}{\mu_{j_{0}}-\mu_{0, j_{0}}}\right)^{2} \geq m^{2} \frac{L^{2}}{4} \geq M m \frac{L^{2}}{4} .
$$

Note further that for $m \in \mathbb{N}$ there are 8 different $\mu \in \mathbb{Z}^{2}$ such that $|\mu|_{\infty}=m$. Hence

$$
\left|F_{\mathrm{q} 3}^{(1)}(z)-F_{\mathrm{q} 3, M}^{(1)}(z)\right| \leq \frac{8 a \exp \left(\frac{\left|k^{2}\right|}{4 a^{2}}-a^{2} z_{3}^{2}-M^{2} L^{2}\right)}{\pi^{3 / 2} a^{2} M L^{2}} \sum_{m=M+1}^{\infty} \exp \left(-2 M L^{2} a^{2}(m-(M+1))\right) .
$$


The assertion now follows using the geometric series.

For $F_{\mathrm{q} 3, M}^{(2, \pm)}$, we define $\mu_{0} \in \mathbb{Z}^{2}$ by $\left|\alpha^{\left(\mu_{0}\right)}\right|_{\infty} \leq\left|\alpha^{(\mu)}\right|_{\infty}$ for all $\mu \in \mathbb{Z}^{2}$ and set

$$
M_{0}=\max \left\{\left|\frac{L_{1} \alpha_{1}}{2 \pi}+\mu_{0,1}\right|,\left|\frac{L_{2} \alpha_{2}}{2 \pi}+\mu_{0,2}\right|\right\} .
$$

We also set

$$
M_{1}=M_{0}+ \begin{cases}\frac{L}{2 \pi} \sqrt{\operatorname{Re}\left(k^{2}\right)}, & \operatorname{Re}\left(k^{2}\right) \geq 0 \\ 0, & \text { otherwise }\end{cases}
$$

Theorem 5.2 Let $\mu_{0}, M_{0}$ and $M_{1}$ be defined as above and let $M \geq M_{1}$ such that also $\operatorname{Im}\left(k \rho_{\mu}\right) \geq$ $\operatorname{Re}\left(k \rho_{\mu}\right)$ for all $\mu \in \mathbb{Z}^{2}$ with $\left|\mu-\mu_{0}\right|_{\infty}>M$. Then

$$
\begin{aligned}
\left|F_{\mathrm{q} 3}^{(2, \pm)}(z)-F_{\mathrm{q} 3, M}^{(2, \pm)}(z)\right| \leq & \frac{\exp \left(\left|\frac{k}{2 a}\right|^{2}-a^{2} z_{3}^{2}-\frac{2 \pi^{2}\left(M-M_{0}\right)}{a^{2} L^{2}}\right)}{\pi \max _{j=1,2} L_{j}\left(1-\exp \left(-\frac{2 \pi^{2}\left(M-M_{0}\right)}{a^{2} L^{2}}\right)\right)} \mathrm{e}^{-\frac{\pi^{2}}{a^{2} L^{2}}\left(M-M_{0}\right)^{2}} \\
& +\frac{\exp \left(-\frac{\sqrt{2} \pi\left|z_{3}\right|}{L}\right)}{\pi \max _{j=1,2} L_{j}\left(1-\exp \left(-\frac{\sqrt{2} \pi\left|z_{3}\right|}{L}\right)\right)} \mathrm{e}^{-\frac{\sqrt{2} \pi}{L}\left|z_{3}\right|\left(M-M_{1}\right)} .
\end{aligned}
$$

If additionally $\pm a z_{3}+\operatorname{Im}\left(k \rho_{\mu} /(2 a)\right) \geq 0$ for all $\mu \in \mathbb{Z}^{2}$ with $\left|\mu-\mu_{0}\right|_{\infty}>M$, then

$$
\left|F_{\mathrm{q} 3}^{(2, \pm)}(z)-F_{\mathrm{q} 3, M}^{(2, \pm)}(z)\right| \leq \frac{\exp \left(\left|\frac{k}{2 a}\right|^{2}-a^{2} z_{3}^{2}-\frac{2 \pi^{2}\left(M-M_{0}\right)}{a^{2} L^{2}}\right)}{\pi \max _{j=1,2} L_{j}\left(1-\exp \left(-\frac{2 \pi^{2}\left(M-M_{0}\right)}{a^{2} L^{2}}\right)\right)} \mathrm{e}^{-\frac{\pi^{2}}{a^{2} L^{2}}\left(M-M_{0}\right)^{2}} .
$$

Proof: In the case $\pm a z_{3}+\operatorname{Im}\left(k \rho_{\mu} /(2 a)\right) \geq 0$ for $\left|\mu-\mu_{0}\right|_{\infty}=m>M$, we use (3.5) to estimate

$$
\begin{aligned}
& \left|F_{\mathrm{q} 3}^{(2, \pm)}(z)-F_{\mathrm{q} 3, M}^{(2, \pm)}(z)\right| \\
& =\frac{1}{4 L_{1} L_{2}}\left|\sum_{m=M+1}^{\infty} \sum_{\left|\mu-\mu_{0}\right|_{\infty}=m} \frac{1}{k \rho_{\mu}} \mathrm{e}^{\mathrm{i} k \alpha^{(\mu)} \cdot z} \mathrm{e}^{\mp \mathrm{i} k \rho_{\mu} z_{3}} \operatorname{erfc}\left( \pm a z_{3}-\mathrm{i} \frac{k \rho_{\mu}}{2 a}\right)\right| \\
& \leq \frac{\exp \left(\left|\frac{k}{2 a}\right|^{2}-a^{2} z_{3}^{2}\right)}{4 L_{1} L_{2}} \sum_{m=M+1}^{\infty} \sum_{\left|\mu-\mu_{0}\right|=m} \frac{1}{\left|k \rho_{\mu}\right|} \exp \left(-\frac{\left|\alpha+q^{(\mu)}\right|^{2}}{4 a^{2}}\right) .
\end{aligned}
$$

As in the proof of Theorem 4.3, we obtain

$$
\left|\alpha+q^{(\mu)}\right|^{2} \geq \frac{4 \pi^{2}}{\max _{j=1,2} L_{j}}\left[\left(M-M_{0}\right)^{2}+2\left(M-M_{0}\right)(m-M)\right]
$$

and for $\operatorname{Im}\left(k \rho_{\mu}\right) \geq \operatorname{Re}\left(k \rho_{\mu}\right)$ as in (4.4) and (4.5) that

$$
\left|k \rho_{\mu}\right|^{2} \geq \frac{4 \pi^{2}}{\max _{j=1,2} L_{j}}\left(m-M_{1}\right)^{2} \geq \frac{4 \pi^{2}}{\max _{j=1,2} L_{j}}(m-M)^{2} .
$$


Hence

$$
\begin{aligned}
& \left|F_{\mathrm{q} 3}^{(2, \pm)}(z)-F_{\mathrm{q} 3, M}^{(2, \pm)}(z)\right| \\
& \leq \frac{L \exp \left(\left|\frac{k}{2 a}\right|^{2}-a^{2} z_{3}^{2}-\frac{\pi^{2}\left(M-M_{0}\right)^{2}}{a^{2} L^{2}}\right)}{\pi L_{1} L_{2}} \sum_{m=M+1}^{\infty} \exp \left(-\frac{2 \pi^{2}}{a^{2} L^{2}}\left(M-M_{0}\right)(m-M)\right) \\
& \leq \frac{\exp \left(\left|\frac{k}{2 a}\right|^{2}-a^{2} z_{3}^{2}-\frac{\pi^{2}\left(M-M_{0}\right)^{2}}{a^{2} L^{2}}\right)}{\pi \max _{j=1,2} L_{j}} \frac{\exp \left(-\frac{2 \pi^{2}\left(M-M_{0}\right)}{a^{2} L^{2}}\right)}{1-\exp \left(-\frac{2 \pi^{2}\left(M-M_{0}\right)}{a^{2} L^{2}}\right)} .
\end{aligned}
$$

To treat the general case we derive as in (4.4) and (4.5) that for $\left|\mu-\mu_{0}\right|_{\infty}=m>M$,

$$
\operatorname{Im}\left(k \rho_{\mu}\right) \geq \frac{\sqrt{2} \pi}{\max _{j=1,2} L_{j}}\left(m-M_{1}\right) .
$$

As in the proof of Theorem 4.4, we obtain an additional series, which we estimate by

$$
\begin{aligned}
\sum_{m=M+1}^{\infty} \sum_{\left|\mu-\mu_{0}\right|_{\infty}=m} \frac{\exp \left(-\operatorname{Im}\left(k \rho_{\mu}\right)\left|z_{3}\right|\right)}{4 L_{1} L_{2}\left|k \rho_{\mu}\right|} & \leq \frac{\max _{j=1,2} L_{j}}{\pi L_{1} L_{2}} \sum_{m=M+1}^{\infty} \exp \left(-\frac{\sqrt{2} \pi}{L}\left(m-M_{1}\right)\left|z_{3}\right|\right) \\
& \leq \frac{1}{\pi L} \exp \left(-\frac{\sqrt{2} \pi}{L}\left(M-M_{1}\right)\left|z_{3}\right|\right) \frac{\exp \left(-\frac{\sqrt{2} \pi\left|z_{3}\right|}{L}\right)}{1-\exp \left(-\frac{\sqrt{2} \pi\left|z_{3}\right|}{L}\right)}
\end{aligned}
$$

\section{Choosing $a$}

To evaluate the periodic Green's function for a given set of parameters using Ewald's method, some value for the free parameter $a$ needs to be chosen. Such a choice should minimise the cost for the evaluation of the function up to a required accuracy. This goal amounts to an a-priori balancing in the number of terms evaluated in the two truncated series.

To be able to judge the costs for the evaluation of the series fairly, as a prerequisite for choosing $a$, the time for an accurate evaluation of each term in should be bounded. For the case of $F_{*}^{(1)}$, this means bounding the time for an efficient evaluation of $F_{\text {aux }}^{(1)}$. To this end, we fix a value of $M$ and prescribe that given an $\varepsilon>0, a$ should be chosen such that

$$
\left(\frac{|k|^{2}}{4 a^{2}(M+1)}\right)^{M+1} \leq \varepsilon
$$




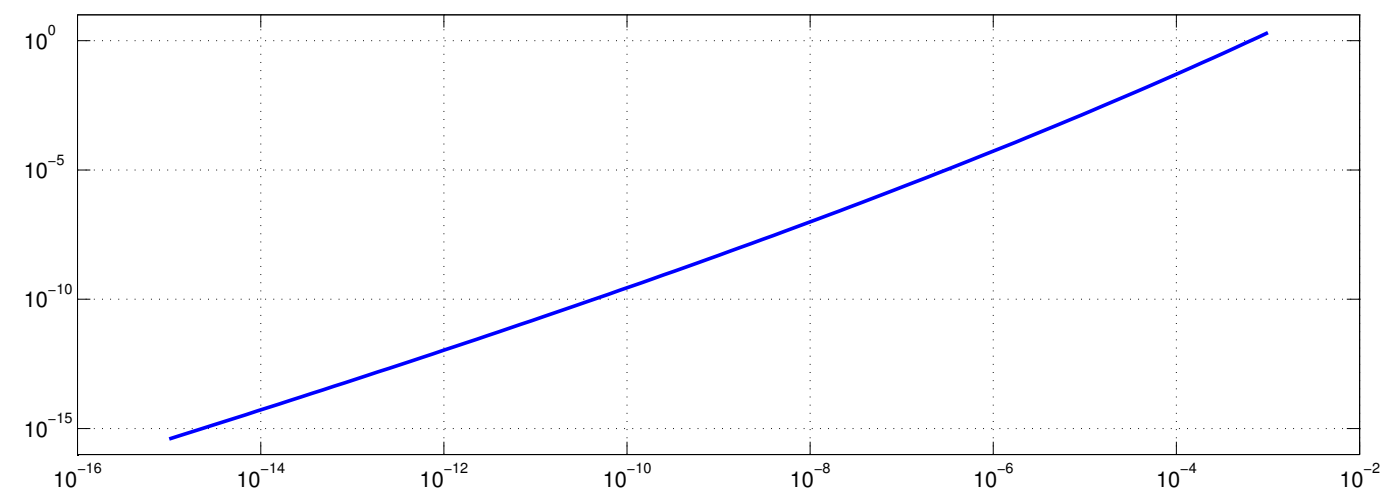

Figure 2: $\varepsilon$ against the right-hand side of (6.2) for $M=15$ and $\nu=1$

which amounts to the lower bound for $a$,

$$
a \geq \frac{|k|}{\sqrt{M+1} \varepsilon^{\frac{2}{M+1}}}
$$

For any $a$ satisfying this lower bound, we have by Lemma 4.1 that

$$
\left|F_{\text {aux }}^{(1)}\left(R, \frac{k^{2}}{4 a^{2}}, \nu\right)-\sum_{j=0}^{M} \frac{1}{j !}\left(\frac{k^{2}}{4 a^{2}}\right)^{j} \int_{1}^{\infty} s^{-j-\nu} \mathrm{e}^{-R s} \mathrm{~d} s\right| \leq \frac{\mathrm{e}^{(M+1) \varepsilon^{1 /(M+1)}} \varepsilon}{M+\nu} .
$$

Figure 2 shows a plot of $\varepsilon$ against the right-hand side of (6.2), demonstrating a good correspondence.

A second issue in choosing $a$ is that care has to be taken to avoid instability and cancellation effects. The bounds on the terms in the series derived in Sections 3-5 all contain a factor $\exp \left(|k|^{2} /\left(4 a^{2}\right)-a^{2} z_{j}^{2}\right), j=2$ or $j=3$. The control parameter $a$ has to be chosen so that this expression is of the same order of magnitude as the expected value of the Green's function to avoid cancellation effects.

Crude estimates of the order of magnitude of the value of the Green's function can be obtained from (2.5) or (2.8). Choosing $\mu_{0}$ as in Theorems 4.2 and 5.1, respectively, one can define

$$
\begin{aligned}
& G_{\mathrm{q} 2, \text { est }}(z)=\frac{\mathrm{i}}{2} \sum_{\mu=\mu_{0}-p}^{\mu_{0}+p} \mathrm{e}^{\mathrm{i} \mu \alpha L} H_{0}^{(1)}\left(k\left|z-\mu\left(\begin{array}{l}
L \\
0
\end{array}\right)\right|\right), \\
& G_{\mathrm{q} 3, \text { est }}(z)=\frac{1}{2 \pi} \sum_{\left|\mu-\mu_{0}\right|_{\infty} \leq p} \mathrm{e}^{\mathrm{i} \alpha \cdot p^{(\mu)}} \frac{\mathrm{e}^{\mathrm{i} k\left|z-p^{(\mu)}\right|}}{\left|z-p^{(\mu)}\right|},
\end{aligned}
$$


for some small value of $p$, i.e. twice the value of truncated series of point sources. The condition

$$
\exp \left(\frac{|k|^{2}}{4 a^{2}}-a^{2} z_{j}^{2}\right) \leq\left|G_{\mathrm{q} j, \text { est }}(z)\right|
$$

can be used for computing a lower bound for sensible values of $a$. Assuming $\left|z_{j}\right|>0$, we obtain

$$
a^{2} \geq \sqrt{\frac{|k|^{2}}{4}+\left(\frac{\log \left|G_{\mathrm{q} j, \text { est }}(z)\right|}{2\left|z_{j}\right|^{2}}\right)^{2}}-\frac{\log \left|G_{\mathrm{q} j, \text { est }}(z)\right|}{2\left|z_{j}\right|^{2}} .
$$

Note, however, that (6.3) cannot be satisfied for any value of $a$ if $z_{j}=0$ and $\left|G_{\mathrm{q} j \text {,est }}(z)\right|<1$. For small values of $\left|z_{j}\right|$ the lower bound (6.4) may also require a value of $a$ far from a value considered optimal for the reasons outlined below. These situations reflect cases in which we can only guarantee an absolute, but not a relative error in the value computed for the Green's function, as individual terms in both of the series in Ewald's expression will be larger than its value. One way to deal with this case is to use special computer arithmetic, but we don not pursue this approach here.

We now return to the question of an optimal choice for $a$, bearing in mind the necessity to satisfy the lower bounds (6.1) and (6.4). Given the complexity of error estimates, the exact optimal $a$ for a given parameter set is hard to obtain. However, the dominating factor influenceable by choosing $a$ in Theorems 4.2 and 5.1 is $\exp \left(-a^{2} L^{2} M^{(1)}{ }^{2}\right)$, where $M^{(1)}$ denotes the cut-off index of the series. Similarly, the dominating factor in both Theorem 4.3 and the second estimate in Theorem 5.2 is $\exp \left(-\frac{-\pi^{2}}{a^{2} L^{2}}\left(M^{(2)}-M_{0}\right)^{2}\right)$. Note that the slower decaying term in Theorem 4.4 and in the first estimate in Theorem 5.2 is independent of $a$.

For the two-dimensional case, minimising $M^{(1)}+M^{(2)}$ while ensuring that both dominating terms are equal to a prescribed value $\varepsilon$ leads to the condition

$$
a=\frac{\sqrt{\pi}}{L}
$$

This is the same value as recommended in (Capolino et al., 2005). An optimal choice of $a$ is thus the maximum of this value and the lower bounds in (6.1) and (6.4).

We have carried out a number of numerical tests to study the effect of this choice of $a$. Figures 3 and 4 in the left-hand column show the effect varying $a$ has on the accuracy of the calculation. The 

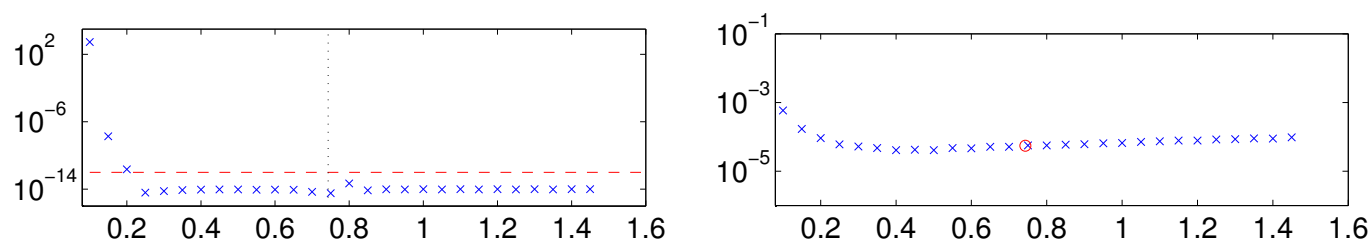

(a) $z_{2}=0.5$
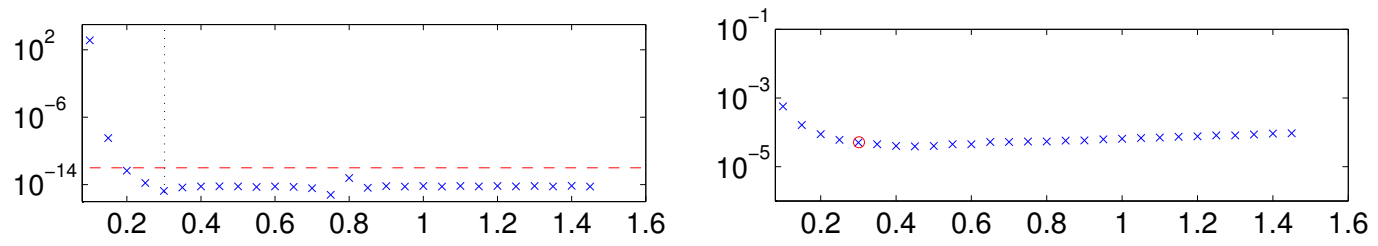

(b) $z_{2}=0.125$
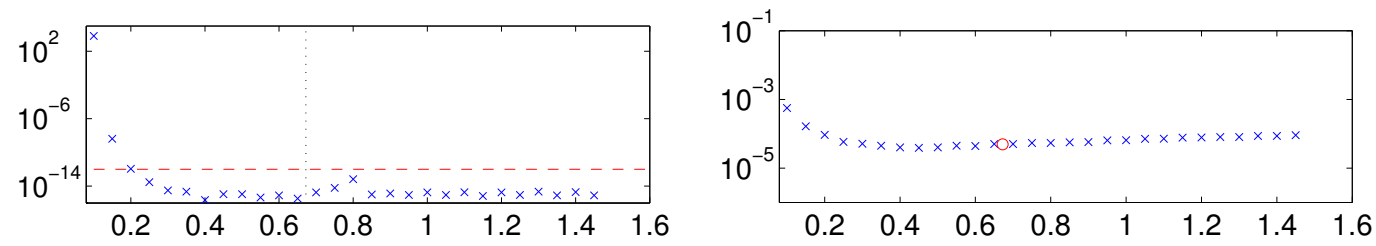

(c) $z_{2}=0$

Figure 3: Plots of difference to value computed for recommended $a$ against $a$ (left) and computation time per Green's function evaluation against $a$ in seconds (right). All examples use $k=\sqrt{2}, z_{1}=0.02$, $\alpha=\sqrt{3} / 4$ and $L=2 \pi$. 

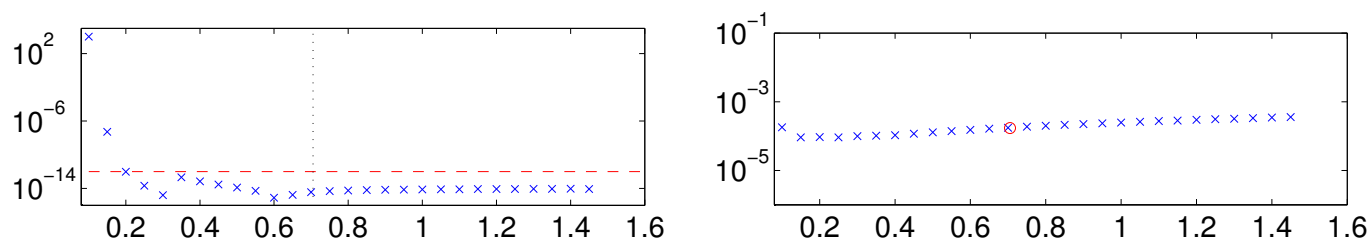

(d) $z_{2}=0.5$
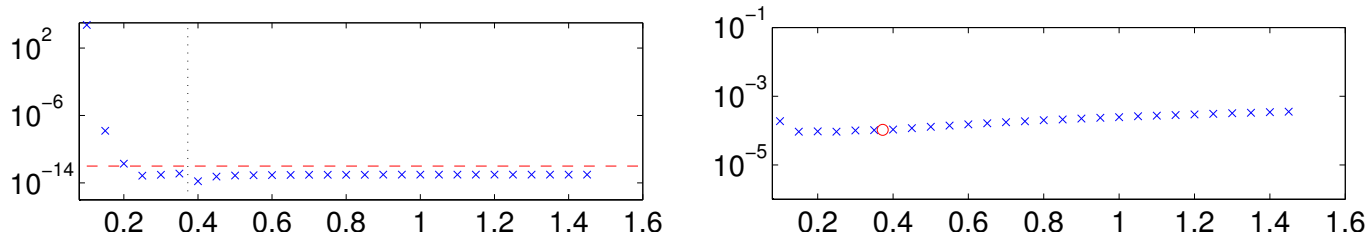

(e) $z_{2}=0.125$
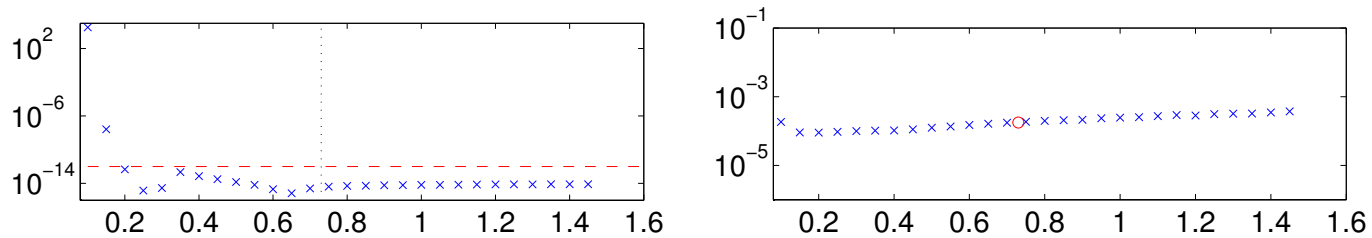

(f) $z_{2}=0$

Figure 4: Plots of difference to value computed for recommended $a$ against $a$ (left) and computation time per Green's function evaluation against $a$ in seconds (right). All examples use $k=\sqrt{2}, z_{1}=0.02$, $\alpha=\sqrt{3} / 4$ and $L=8 \pi$.

distance from the horizontal line $z_{2}=0$ is different from picture to picture while all other parameters stay constant. Figure 3 uses a period of $2 \pi$ while Figure 4 uses one of $8 \pi$. Partial sums of the series representing the Green's functions were calculated up to a point where the estimates given in the previous sections guarantee an absolute error of less than $10^{-12}$, indicated by the red dashed line. The dotted vertical line signifies the location of the recommended value of $a$. The plotted accuracy corresponds to the absolute difference to the value obtained for the recommended value of $a$. In all cases, our recommended value stays well clear of the range of $a$ where cancellation effects occur and the calculation becomes unstable.

On the right hand column of both figures, the effect that varying $a$ has on the computational time is displayed. The red circle indicates the location and computational time for the recommended value of $a$. The computational time required for this $a$ is never significantly higher than the minimal 
time among all calculations. However, for the two-dimensional Green's function, the effect of varying $a$ on computational time is not very pronounced.

For the three-dimensional case, balancing the costs of the series evaluations is more complicated, as these costs grow quadratically in $M^{(l)}, l=1,2$. Thus, the value of $M_{0}$ becomes important. Again equating the dominating terms to $\varepsilon$, we minimse

$$
M^{(1)^{2}}+M^{(2)^{2}}=\frac{\log (1 / \varepsilon)}{a^{2} L^{2}}+\left(\frac{a L \sqrt{\log (1 / \varepsilon)}}{\pi}+M_{0}\right)^{2} .
$$

This leads to the equation

$$
a^{4}+\frac{M_{0} \pi}{L \sqrt{\log (1 / \varepsilon)}} a^{3}-\frac{\pi^{2}}{L^{4}}=0
$$

The left-hand side is a strictly monotonic function for positive $a$ and has a single root. We recommend to choose $a$ as the maximum of this root and the lower bounds in (6.1) and (6.4).

Results analogous to those for the two-dimensional case are presented in Figure 5. The overall results are quite similar, though the effect of choosing $a$ on the total cost of the evaluation is much more pronounced. The recommended value does again not lead to significantly higher costs than the minimal value obtained using these tests. More results along these lines are presented in (Arens, 2010).

Having given an answer to the question of the choice of $a$, recommendations should also be given on when to use Ewald's representation rather than a different one. The obvious alternative is the modal representation which consists only of functions that are relatively simple to evaluate, and it is exponentially convergent for $z_{2} \neq 0$ and $z_{3} \neq 0$, respectively.

Tests have been carried out for the two-dimensional case for various values of $k, L$ and $z_{2}$ comparing computation times. For each combination of parameters, 1000 (for small values of $z_{2}$ ) or 5000 (for large values of $z_{2}$ ) random values of $z_{1}$ were generated. The Green's function was evaluated both using Ewald's representation with the recommended value of $a$ and using the modal representation (2.7). In the case of Ewald's representation, the series were evaluated up to a guaranteed error of $10^{-12}$ using the bounds in Theorems 4.2, 4.3 and 4.4. For the modal representation, the 

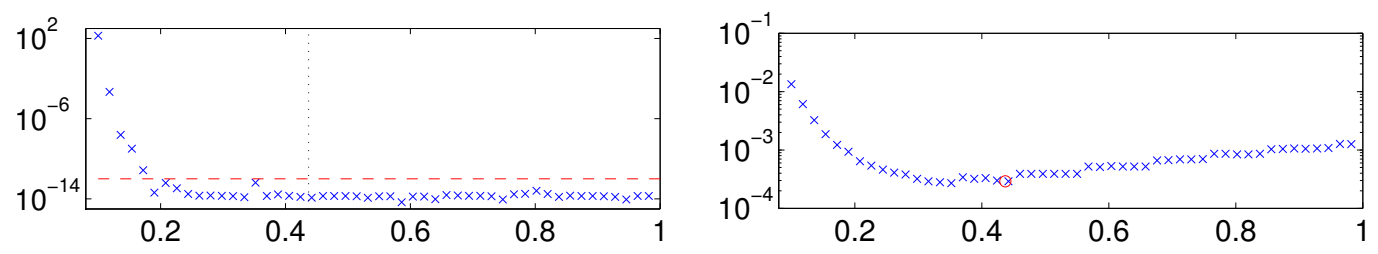

(a) $z_{3}=0.5$
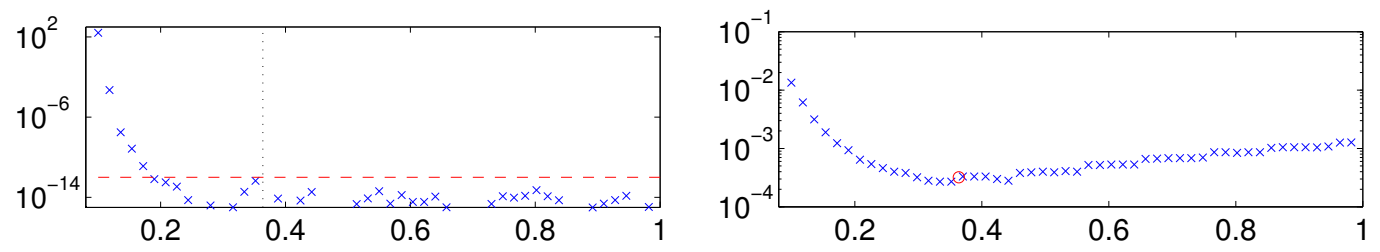

(b) $z_{3}=0.125$
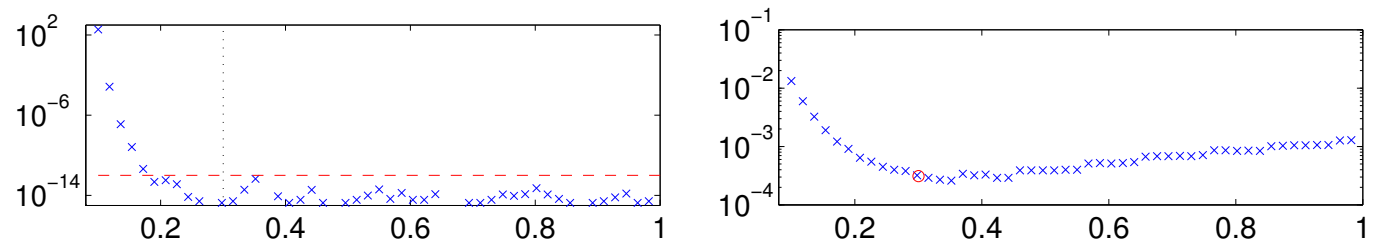

(c) $z_{3}=0$

Figure 5: Plots of difference to value computed for recommended $a$ against $a$ (left) and computation time per Green's function evaluation against $a$ in seconds (right). All examples use $k=\sqrt{2}, z_{1}=0$, $z_{2}=0.02, \alpha_{1}=\alpha_{2}=\sqrt{3} / 4$ and $L_{1}=L_{2}=2 \pi$. 
first partial sum having a value within a $10^{-11}$-neighborhood of the value computed using Ewald's method was evaluated. This methodology contains a bias towards the modal representation in that the modal representation is computed to a slightly less accurate level, that no error estimators need to be computed and that there are no influences due to a possibly pessimistic error estimator.

The results are displayed in Figure 6. Firstly, it appears that the computation times using Ewald's representation remain fairly constant when $z_{1}$ is varied. In contrast, for small $z_{2}$, the computation time using the modal expansion roughly increases by a factor of 2 if $z_{2}$ is divided by 2 . This increase is observable in all examples, uniformly for all parameter choices. For large $z_{2}$, the computation time using the modal expansion also becomes constant. This behaviour corresponds to the fact that for large $z_{2}$ only the plane wave terms form an observable contribution to the Green's function value.

Ewald's method is more efficient only in a small neighborhood of the $z_{2}$ axis. At larger distance, the evaluation is more costly than the modal expansion by a factor ranging between 10 and 50 . However, it must be noted that points with a distance of less than 0.16 from the origin, where the singularity is located, were excluded from being chosen for evaluation as the modal expansion did not reliably converge for such points. Our conclusion is to recommend the use of Ewald's method for points close to the $z_{2}$-axis. A value of $\left|z_{2}\right|=0.2$ seems to be a reasonable choice.

The observations are similar but much more pronounced for the three-dimensional case. Corresponding numerical results are presented in (Arens, 2010).

\section{$7 \quad$ Evaluation of Derivatives}

Applications such as boundary integral equation methods also require the evaluation of derivatives of the Green's functions. Lemmas 3.1 and 3.2 show that the series representing $F_{\mathrm{q} 2}^{(1)}$ and $F_{\mathrm{q} 2}^{(2, \pm)}$ converge uniformly on compact subsets of $\mathbb{R}^{2}$. Hence, expressions for partial derivatives can be obtained by term by term differentiation of these series. The same arguments hold for $F_{\mathrm{q} 3}^{(1)}$ and $F_{\mathrm{q} 3}^{(2, \pm)}$. Also 


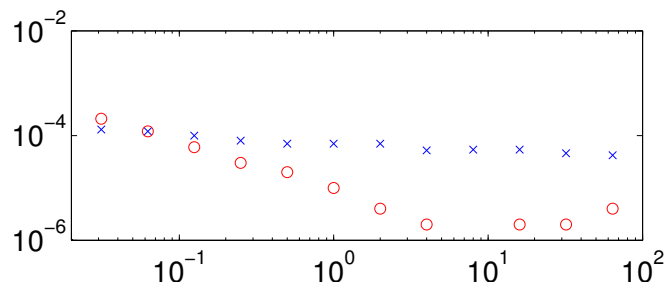

(a) $k=\sqrt{2}, L=2 \pi$

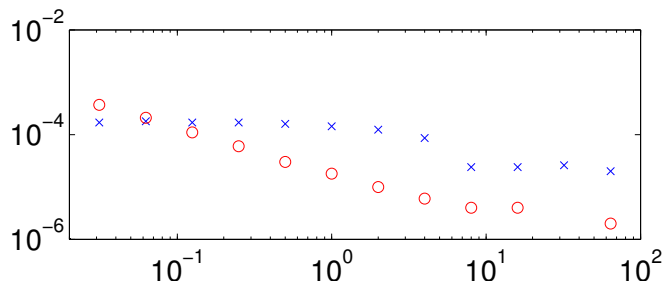

(b) $k=\sqrt{2}, L=4 \pi$

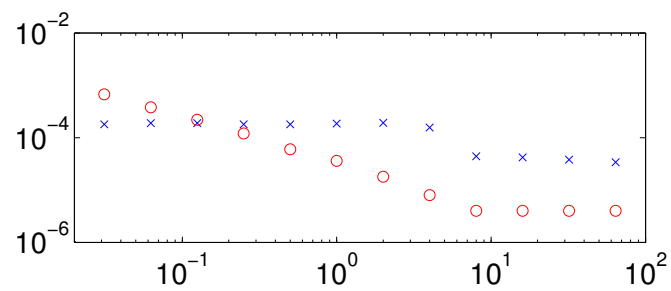

(c) $k=\sqrt{2}, L=8 \pi$

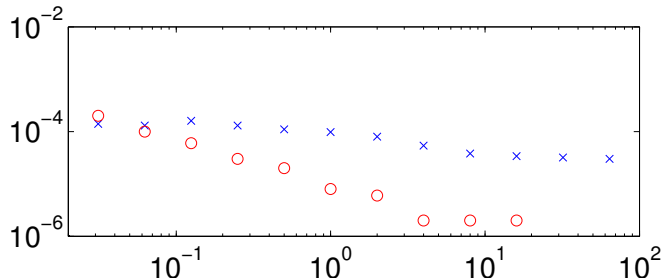

(d) $k=2 \sqrt{2}, L=2 \pi$

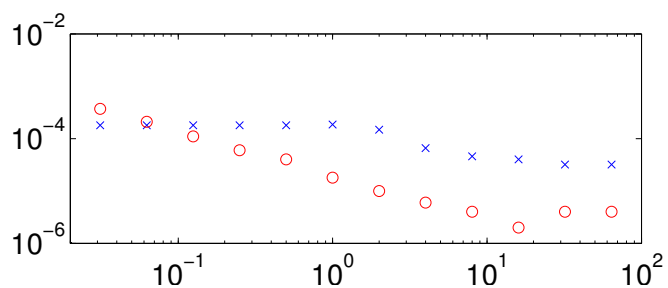

(e) $k=2 \sqrt{2}, L=4 \pi$

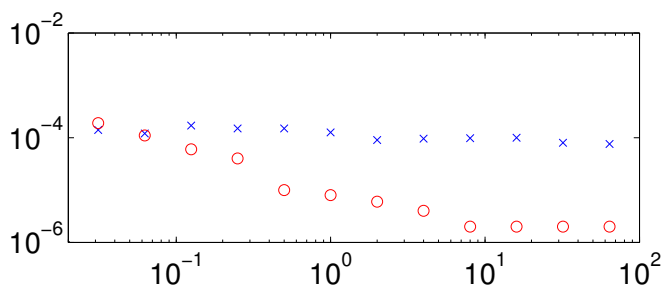

(f) $k=4 \sqrt{2}, L=2 \pi$

Figure 6: Plots of computation time against $z_{2}$ for Ewald's method using the recommended value of $a$ (blue crosses) and for the modal expansion (red circles). Between 1000 and 5000 random values for $z_{1}$ are used and computation times are averaged. Values using Ewald's representation are computed up to a guaranteed error of $10^{-12}$, values using the modal expansion up to a difference of $10^{-11}$ to the value for Ewald's method. All examples use $\alpha=\sqrt{3} / 4$. 
noting

$$
\frac{\partial}{\partial R} F_{\text {aux }}^{(1)}(R, \kappa, \nu)=-F_{\text {aux }}^{(1)}(R, \kappa, \nu-1) \quad \text { for } \nu \geq 1
$$

we obtain the expressions

$$
\begin{aligned}
\nabla F_{\mathrm{q} 2}^{(1)}(z)=-\frac{a^{2}}{2 \pi} \sum_{\mu \in \mathbb{Z}} \mathrm{e}^{\mathrm{i} \mu \alpha L}\left(z-\mu L e_{1}\right) F_{\text {aux }}^{(1)}\left(a^{2}\left|z-\mu L e_{1}\right|^{2},\left(\frac{k}{2 a}\right)^{2}, 0\right), \\
\nabla F_{\mathrm{q} 2}^{(2, \pm)}(z)=\frac{\mathrm{i}}{4 L} \sum_{\mu \in \mathbb{Z}} \frac{1}{k \beta_{\mu}} \mathrm{e}^{\mathrm{i} k \alpha_{\mu} z_{1}} \mathrm{e}^{\mp 1 k \beta_{\mu} z_{2}}\left[\left(\begin{array}{c}
\mathrm{i} k \alpha_{\mu} \\
\mp \mathrm{i} k \beta_{\mu}
\end{array}\right) \operatorname{erfc}\left( \pm a z_{2}-\mathrm{i} \frac{k \beta_{\mu}}{2 a}\right)\right. \\
\left.-\frac{2}{\sqrt{\pi}}\left(\begin{array}{c}
0 \\
\pm a
\end{array}\right) \exp \left(-\left( \pm a z_{2}-\mathrm{i} \frac{k \beta_{\mu}}{2 a}\right)^{2}\right)\right],
\end{aligned}
$$

as well as

$$
\begin{aligned}
& \nabla F_{\mathrm{q} 3}^{(1)}(z)=-\frac{a^{3}}{2 \pi^{3 / 2}} \sum_{\mu \in \mathbb{Z}^{2}} \mathrm{e}^{\mathrm{i} \alpha \cdot p^{(\mu)}}\left(z-p^{(\mu)}\right) F_{\mathrm{aux}}^{(1)}\left(a^{2}\left|z-p^{(\mu)}\right|^{2},\left(\frac{k}{2 a}\right)^{2},-\frac{1}{2}\right), {\left[\begin{array}{c}
\mathrm{i} k \alpha_{1}^{(\mu)} \\
\mathrm{i} k \alpha_{2}^{(\mu)} \\
\mp \mathrm{i} k \rho_{\mu}
\end{array}\right) \operatorname{erfc}\left( \pm a z_{3}-\mathrm{i} \frac{k \rho_{\mu}}{2 a}\right) } \\
&\left.\nabla F_{\mathrm{q} 3}^{(2, \pm)}(z)=\frac{\mathrm{i}}{4 L_{1} L_{2}} \sum_{\mu \in \mathbb{Z}^{2}} \frac{1}{k \rho_{\mu}} \mathrm{e}^{\mathrm{i} k \alpha^{(\mu)} \cdot z} \mathrm{e}^{\mp \mathrm{i} k \rho_{\mu} z_{3}}\left(\begin{array}{c}
0 \\
0 \\
0
\end{array}\right) \exp \left(-\left( \pm a z_{3}-\mathrm{i} \frac{k \rho_{\mu}}{2 a}\right)^{2}\right)\right] . \\
&\left.-\frac{2}{\sqrt{\pi}}\left(\begin{array}{c} 
\pm a
\end{array}\right)\right]
\end{aligned}
$$

For the remainder of this section we will only treat the 2D case. The biperiodic functions are easy to treat in the same way.

Truncated versions of the series denoted by $\nabla F_{\mathrm{q} 2, M}^{(1)}$ and $\nabla F_{\mathrm{q} 2, M}^{(2, \pm)}$ are defined by replacing the series in the expressions above by summations $\sum_{\mu=\mu_{0}-M}^{\mu_{0}+M}$. The following theorems give estimates for the remainders.

Theorem 7.1 Define $\mu_{0} \in \mathbb{Z}$ by $z_{1}-\mu_{0} L \in(-L / 2, L / 2]$ and let $M \in \mathbb{N}$. Then there holds

$$
\left\|\nabla F_{\mathrm{q} 2}^{(1)}(z)-\nabla F_{\mathrm{q} 2, M}^{(1)}(z)\right\|_{\infty} \leq \frac{\exp \left(|k|^{2} /\left(4 a^{2}\right)-a^{2} z_{2}^{2}\right)}{\pi \sqrt{(M L)^{2}+z_{2}^{2}}\left(1-\exp \left(-2 a^{2} M L^{2}\right)\right)} \mathrm{e}^{-(a M L)^{2}} .
$$


Proof: Exactly as in the proof of Theorem 4.2, we estimate

$$
\begin{aligned}
\left\|\nabla F_{\mathrm{q} 2}^{(1)}(z)-\nabla F_{\mathrm{q} 2, M}^{(1)}(z)\right\|_{\infty} & \leq \frac{a^{2} \exp \left(|k|^{2} /(2 a)^{2}-a^{2} z_{2}^{2}\right)}{2 \pi} \sum_{\left|\mu-\mu_{0}\right| \geq M+1} \frac{\exp \left(-a^{2}\left(z_{1}-\mu L\right)^{2}\right)}{a^{2} \sqrt{\left(z_{1}-\mu L\right)^{2}+z_{2}^{2}}} \\
& \leq \frac{\exp \left(|k|^{2} /(2 a)^{2}-a^{2} z_{2}^{2}\right)}{\pi} \frac{\exp \left(-a^{2}(M L)^{2}\right)}{\sqrt{(M L)^{2}+z_{2}^{2}}} \sum_{\mu=M+1}^{\infty} \mathrm{e}^{-2 M L^{2} a^{2}(\mu-(M+1))} .
\end{aligned}
$$

A geometric series argument then gives the assertion.

Theorem 7.2 Let the assumptions of Theorem 4.3 hold and, in addition, let $K$ be defined by

$$
K= \begin{cases}\max _{\mu \in \mathbb{Z}}\left|1-1 / \alpha_{\mu}^{2}\right|^{-1 / 2}, & \text { if } \operatorname{Re}\left(k^{2}\right)>\operatorname{Im}\left(k^{2}\right) \\ 1, & \text { otherwise. }\end{cases}
$$

Then

$$
\begin{aligned}
& \| \nabla F_{\mathrm{q} 2}^{(2, \pm)}(z)- \nabla F_{\mathrm{q} 2, M}^{(2, \pm)}(z) \|_{\infty} \\
& \leq\left(K+\frac{2 a}{\sqrt{\pi} \min \operatorname{Im}\left(k \beta_{\mu_{0} \pm M}\right)}\right) \frac{\exp \left(\left|\frac{k}{2 a}\right|^{2}-a^{2} z_{2}^{2}-\frac{2 \pi^{2}\left(M-M_{0}\right)}{a^{2} L^{2}}\right)}{2 L\left(1-\exp \left(-\frac{2 \pi^{2}\left(M-M_{0}\right)}{a^{2} L^{2}}\right)\right)} \mathrm{e}^{-\frac{\pi^{2}}{a^{2} L^{2}}\left(M-M_{0}\right)^{2}} .
\end{aligned}
$$

Proof: We start with the reformulation

$$
\left\|\nabla F_{\mathrm{q} 2}^{(2, \pm)}(z)-\nabla F_{\mathrm{q} 2, M}^{(2, \pm)}(z)\right\|_{\infty}=\frac{1}{4 L}\left\|\sum_{\left|\mu-\mu_{0}\right|>M} \frac{1}{k \beta_{\mu}} \mathrm{e}^{\mathrm{i} k \alpha_{\mu} z_{1}}\left[\left(\begin{array}{c}
\mathrm{i} k \alpha_{\mu} \\
\mp \mathrm{i} k \beta_{\mu}
\end{array}\right) T_{1, \mu}^{ \pm}+\left(\begin{array}{c}
0 \\
\pm a
\end{array}\right) T_{2, \mu}^{ \pm}\right]\right\|_{\infty}
$$

where

$$
T_{1, \mu}^{ \pm}=\mathrm{e}^{\mp \mathrm{i} k \beta_{\mu} z_{2}} \operatorname{erfc}\left( \pm a z_{2}-\mathrm{i} \frac{k \beta_{\mu}}{2 a}\right), \quad T_{2, \mu}^{ \pm}=\frac{2}{\sqrt{\pi}} \mathrm{e}^{\mp \mathrm{i} k \beta_{\mu} z_{2}} \exp \left(-\left( \pm a z_{2}-\mathrm{i} \frac{k \beta_{\mu}}{2 a}\right)^{2}\right)
$$

Observing that

$$
\left|\frac{\alpha_{\mu}}{\beta_{\mu}}\right|=\left|\frac{1}{1-\frac{1}{\alpha_{\mu}^{2}}}\right|^{1 / 2} \leq K, \quad \mu \in \mathbb{Z}
$$

we conclude

$$
\left\|\nabla F_{\mathrm{q} 2}^{(2, \pm)}-\nabla F_{\mathrm{q} 2, M}^{(2, \pm)}\right\|_{\infty} \leq \frac{1}{4 L} \sum_{\left|\mu-\mu_{0}\right|>M}\left(K\left|T_{1, \mu}^{ \pm}\right|+\frac{a}{\left|k \beta_{\mu}\right|}\left|T_{2, \mu}^{ \pm}\right|\right) .
$$

Similar to the argument in the proof of Theorem 4.3, we estimate $\left|T_{1, \mu}^{ \pm}\right|$by

$$
\left|T_{1, \mu}^{ \pm}\right| \leq \exp \left(\left|\frac{k}{2 a}\right|^{2}-a^{2} z_{2}^{2}\right) \exp \left(-\frac{(\alpha+2 \pi \mu / L)^{2}}{4 a^{2}}\right) \text {. }
$$



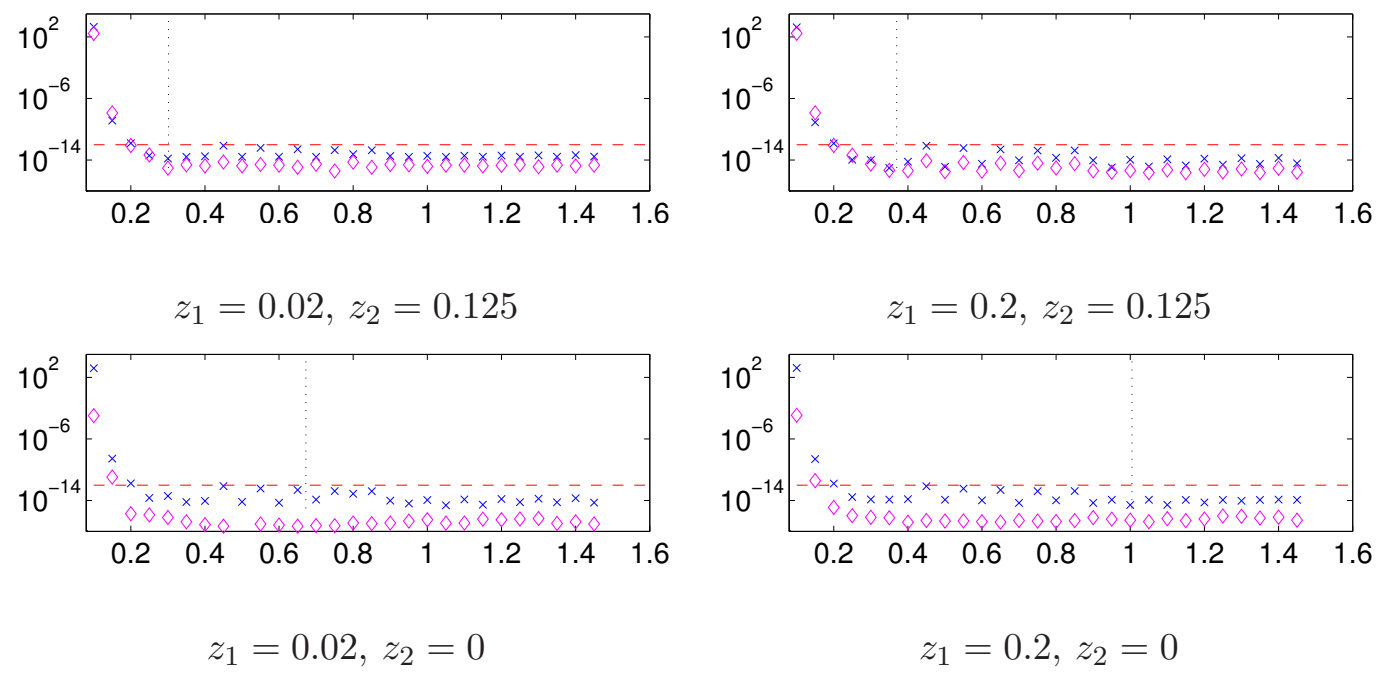

Figure 7: Plots of difference to value computed for recommended $a$ against $a$ for evaluation of the Green's function's gradient. Blue crosses indicate the derivative with respect to $z_{1}$, magenta diamonds those with respect to $z_{2}$. All examples use $k=\sqrt{2}, \alpha=\sqrt{3} / 4$ and $L=2 \pi$.

Obviously, $\left|T_{2, \mu}^{ \pm}\right|$can be bounded by

$$
\left|T_{2, \mu}^{ \pm}\right| \leq \frac{2}{\sqrt{\pi}}\left|\exp \left(\left(\frac{k \beta_{\mu}}{2 a}\right)^{2}-a^{2} z_{2}^{2}\right)\right| \leq \frac{2}{\sqrt{\pi}} \exp \left(\left|\frac{k}{2 a}\right|^{2}-a^{2} z_{2}^{2}\right) \exp \left(-\frac{(\alpha+2 \pi \mu / L)^{2}}{4 a^{2}}\right) .
$$

Inserting these bounds in the reformulation above and rearranging terms yields

$$
\begin{aligned}
&\left\|\nabla F_{\mathrm{q} 2}^{(2, \pm)}(z)-\nabla F_{\mathrm{q} 2, M}^{(2, \pm)}(z)\right\|_{\infty} \leq\left(K+\frac{2 a}{\sqrt{\pi} \min \operatorname{Im}\left(k \beta_{\mu_{0} \pm M}\right)}\right)\left[\frac{1}{4 L} \exp \left(\left|\frac{k}{2 a}\right|^{2}-a^{2} z_{2}^{2}\right)\right. \\
&\left.\times \sum_{\left|\mu-\mu_{0}\right|>M} \exp \left(-\frac{(\alpha+2 \pi \mu / L)^{2}}{4 a^{2}}\right)\right] .
\end{aligned}
$$

We now derive the assertion by arguing as in the proof of Theorem 4.3.

The considerations for the choice of $a$ remain the same for the evaluation of the gradient. Figure 7 displays some results for computing the partial derivatives. In all computations a guaranteed accuracy of $10^{-12}$ (indicated by the dashed horizontal line) was prescribed and achieved except for those values of $a$ where cancellation errors dominate. The recommended value of $a$ is denoted by the dotted vertical line. Results for computational times are also very similar to those for computing the Green's function itself and hence we omit presenting them.

An estimate analogous to that given in Theorem 4.4 can also be established for the derivatives of 
$F_{\mathrm{q} 2}^{(2, \pm)}$. However, the principles being clear from what has been shown, we do not present the details here.

\section{References}

Abramowitz, M. \& Stegun, I. (1965) Handbook of mathematical functions, unabridged and unaltered republ. of the 1964 edn. Dover Publ.

Arens, T. (1999) The scattering of plane elastic waves by a one-dimensional periodic surface. Math. Meth. Appl. Sci., 22, 55-72.

Arens, T. (2010) Scattering by biperiodic layered media: The integral equation approach. Habilitation Thesis, Karlsruhe Institute of Technology.

Capolino, F., Wilton, D. R. \& Johnson, W. A. (2005) Efficient computation of the 2-D Green's function for 1-D periodic structures using the Ewald method. IEEE Trans. Ant. Prop., 53, $2977-2984$.

Ewald, P. P. (1921) Die Berechnung optischer und elektrostatischer Gitterpotentiale. Ann. Phys., 64, 253-287.

Hopf, E. (1934) Mathematical Problems of Radiative Equilibrium. Cambridge Tracts in Mathematics and Mathematical Physics. Cambridge University Press.

Jordan, K. E., Richter, G. R. \& Sheng, P. (1986) An efficient numerical evaluation of the Green's function for the Helmholtz operator on periodic structures. J. Comput. Phys., 63, 222-235.

Kambe, K. (1967) Theory of electron diffraction by crystals. Green's function and integral equation. Z. Naturforschg., 22a, 422-431.

Kurkcu, H. \& Reitich, F. (2009) Stable and efficient evaluation of periodized Green's functions for the Helmholtz equation at high frequencies. Journal of Computational Physics, 228(1), 75-95. 
Kustepeli, A. \& Martin, A. Q. (2000) On the Splitting Parameter in the Ewald Method. IEEE Transactions on Microwave and Guided Wave Letters, 10(5), 168-170.

Linton, C. M. (1998) The Green's function for the two-dimensional Helmholtz equation in periodic domains. J. Eng. Math., 33, 377-402.

Mathis, A. W. \& Peterson, A. F. (1996) A comparison of acceleration procedures for the two-dimensional periodic green's function. IEEE Trans. Ant. Prop., 44, 567-571.

Sadov, S. Y. (1997) Computation of quasiperiodic fundamental solution of Helmholtz equation. Advances in Difference Equations: Proceedings of the Second International Conference on Difference Equations (Veszprém, 1995). CRC Press, pp. 551-558.

SAnDForT, K. (2010) The Factorization Method for inverse scattering from periodic inhomogeneous media. Ph.D. thesis, Karlsruhe Institute of Technology (KIT). 